\title{
Dragonflies and Damselflies in a region of the Triângulo Mineiro, Minas Gerais: checklist and taxonomic additions
}

\author{
Henrique Venâncio $^{1 *}{ }^{(\mathrm{D})}$, Diogo Silva Vilela ${ }^{(\mathrm{D})}$,Marcela Silva Barbosa $^{3}$ \& Jean Carlos Santos ${ }^{4}$ \\ ${ }^{1}$ Universidade de São Paulo, Faculdade de Filosofia, Ciências e Letras de Ribeirão Preto, Programa de \\ Pós-Graduação em Entomologia, Ribeirão Preto, SP, Brasil. \\ ${ }^{2}$ Autonomous researcher, Rua Jaime Bilharinho, 575, CEP 38065-280, Uberaba, MG, Brasil. \\ ${ }^{3}$ Universidade Federal de Uberlândia, Instituto de Biologia, Uberlândia, MG, Brasil. \\ ${ }^{4}$ Universidade Federal de Sergipe, Departamento de Ecologia, São Cristóvão, SE, Brasil. \\ *Corresponding author: henrivens@gmail.com,
}

VENÂNCIO, H., VILELA, D.S., BARBOSA, M.S., SANTOS, J.C. Dragonflies and Damselflies in a region of the Triângulo Mineiro, Minas Gerais: checklist and taxonomic additions. Biota Neotropica 21(3): e20201182. https://doi.org/ 10.1590/1676-0611-BN-2020-1182

\begin{abstract}
Remaining freshwater systems are historically under threat mainly due to human activities such as agriculture and urbanization. The consequences of such activities are innumerous, and among them there is a decrease of suitable habitats for threatened fauna. In the Brazilian Cerrado, the odonatofauna of palm swamps and riparian forests are still poorly explored, a fact that difficult conservation efforts of the group. Thus, we performed an inventory in several urban and rural sites containing these phytophysiognomies in Uberlândia, Triângulo Mineiro region, western Minas Gerais state. In total, we found 101 Odonata species, seven families and 46 genera in the municipality, with 76 and 66 species, respectively, belonging to palm swamp and forest sites. From this diversity, eight species were first records in the state of Minas Gerais: Neuraeschna claviforcipata Martin, 1909, Phyllocycla cf. medusa Belle, 1988, Diastatops intensa Montgomery, 1940, Oligoclada pachystigma Karsch, 1890, O. xanthopleura Borror, 1931, Angelagrion nathaliae Lencioni, 2008, Telebasis sanguinalis Calvert, 1909 and Telebasis simulacrum (Calvert, 1909). We also sampled Erythrodiplax ana Guillermo-Ferreira \& Vilela 2016, a species listed as endangered (EN) by the IUCN red list. Additionally, we include some taxonomic notes of Forcepsioneura machadorum females, a newly discovered species in the region. Our results contribute to the Odonata database in Brazil and highlights the importance inventories in poorly explored aquatic ecosystems.

Keywords: Odonata; Cerrado; Brazil; inventory; female description.
\end{abstract}

\section{Libélulas de uma região do Triângulo Mineiro, Minas Gerais: lista de espécies e adições taxonômicas}

Resumo: Os sistemas remanescentes de água doce estão historicamente ameaçados, principalmente devido às atividades humanas, como agricultura e urbanização. As consequências de tais atividades são inúmeras, e entre elas há a diminuição de habitats adequados para a fauna ameaçada. No cerrado brasileiro, a odonatofauna de veredas e matas ripárias ainda são pouco exploradas, fato que dificulta os esforços de conservação do grupo. Assim, realizamos um inventário em diversos pontos urbanos e rurais com essas fitofisionomias em Uberlândia, na região do Triângulo Mineiro, oeste de Minas Gerais. No total, foram encontradas 101 espécies de Odonata, sete famílias e 46 gêneros no município, com 76 e 66 espécies, respectivamente, pertencentes a pontos de veredas e de matas. Dessa diversidade, oito espécies foram os primeiros registros no estado de Minas Gerais: Neuraeschna claviforcipata Martin, 1909, Phyllocycla cf. medusa Belle, 1988, Diastatops intensa Montgomery, 1940, Oligoclada pachystigma Karsch, 1890, O. xanthopleura Borror, 1931, Angelagrion nathaliae Lencioni, 2008, Telebasis sanguinalis Calvert, 1909 e Telebasis simulacrum (Calvert, 1909). Nós também coletamos Erythrodiplax ana Guillermo-Ferreira \& Vilela 2016, uma espécie listada como em perigo (EN) pela lista vermelha da IUCN. Além disso, incluímos algumas notas taxonômicas de fêmeas de Forcepsioneura machadorum, uma espécie recém-descoberta na região. Nossos resultados contribuem para o banco de dados Odonata no Brasil e destacam a importância dos inventários em ecossistemas aquáticos pouco explorados.

Palavras-chave: Odonata; Cerrado; Brasil; inventário; descrição de fêmea. 


\section{Introduction}

Freshwater systems comprise a large part of the planet's biodiversity, although being also some of the most vulnerable ecosystems in the current global biodiversity crisis (Albert et al. 2020). The anthropic effects (e.g., deforestation, waste deposit and exotic species) in these environments modify aquatic and terrestrial integrity, endangering future populational stability of several species (Calvão et al. 2016, Reid et al. 2019, Araújo et al. 2020). Thus, to mitigate these impacts, it is important to fill biodiversity gaps as an initial step for determining conservationist actions in aquatic ecosystems. Surveys have helped to fill gaps for many freshwater organisms (Troia \& McManamay 2017, Bolpagni et al. 2018, Guerra et al. 2020) as species distribution and cataloging (Wallacean and Linnean shortfalls, respectively) (Hórtal et al. 2015). However, these shortfalls are still predominant for some threatened and little-known groups, such as aquatic insects (SánchezBayo \& Wyckhuys 2019).

Odonata compose a small portion of aquatic insect, totalizing 6,300 species (Paulson \& Schorr 2020) that occupies freshwater habitats associated to different vegetation types in almost all continents (Corbet 1980). Brazil holds circa of $30 \%$ of this diversity, with approximately 900 described species, being more than $20 \%$ of this number in endemism (Pinto 2020). Many odonate species are known to be endangered with the overexploitation in many regions of the country (ICMBio 2018, Araújo et al. 2020). This situation has encouraged great effort in preservation measures in the last years, including inventories in priority regions (e.g., Cerrado) and species conservation categorizations (De Marco \& Viana 2005, Koroiva et al. 2017, Rodrigues \& Roque 2017, Dalzochio et al. 2018a, Bastos et al. 2019, Garcia Junior et al. 2021, Koroiva et al. 2020, Vilela et al. 2020a). Despite this progress, distribution and taxonomic gaps are still frequently reported problems in the literature, even in explored regions. For example, more than one third of odonate fauna from Serra da Bodoquena and some regions of southeast and west of Minas Gerais could not be classified in an IUCN red list category due to data deficiency (Koroiva et al. 2017, Ávila Júnior et al. 2020, Vilela et al. 2020a).

The majority of odonate studies are concentrated in the Cerrado, one of the Brazilian hotspots (Myers et al. 2000, Miguel et al. 2017). Nonetheless, new recent taxonomic and distribution discoveries have been indicating gaps in little explored and endangered phytophysiognomies of this biome, such as riparian forest (including gallery forest) and veredas (i.e., palm swamps) (Rodrigues et al. 2018, Barbosa et al. 2019, Palacio et al. 2020, Lozano \& Rodrigues 2018). Both formations are associated with important water courses to local and regional biodiversity and hydrographic maintenance (Ribeiro \& Walter 1998, Latrubesse et al. 2019). Despite this similarity, these environments differ in aquatic and terrestrial characteristics, including plant community composition (Oliveira-Filho \& Ratter 2002). Water bodies of palm swamps are mainly composed by a variety of macrophytes species surrounded by terrestrial grassy-shrubby plants, generally associated to "buriti" palms [Mauritia flexuosa L.f. (Arecaceae)], while large canopy trees cover riparian zones in forests (Ribeiro \& Walter 1998, Araújo et al. 2002, Oliveira-Filho \& Ratter 2002). These characteristics, including aquatic types (i.e., lotic and lentic systems), are determinant for the diversity of dragonflies of the Cerrado. For example, the low solar radiation in extensive areas of closed canopy riparian forests is essential for adults of small size Zygoptera (De Marco et al. 2015), while open palm swamps favor the presence of endemic macrophytes (Araújo et al. 2002), which are sites for oviposition and development of some heliothermic dragonflies (Vilela et al. 2016, Brito et al. 2020). In relation to types of aquatic systems, lotic and lentic environments have unique physical-chemical, biotic and geographic distribution characteristics that have resulted in distinct dragonfly diversifications of these habitats during the evolutionary history of the group (Letsch et al. 2016). Consequently, the composition of species between lotic and lentic bodies of water are distinct, differing at broader taxonomic levels, such as family (Vilela et al. 2020a, Pires et al. 2019). Such habitat heterogeneity is known to harbor different Odonata communities (Dutra \& De Marco 2015), reflecting in great species diversity and endemism (Calvert 1909, Guillermo-Ferreira et al. 2016, Vilela et al. 2016, 2018).

Despite the habitat heterogeneity, the diversity of palm swamps and riparian forests are highly threatened by human activities (Sano et al. 2010, Colli et al. 2020). Modifications generated by land use, such as monoculture, livestock and urban areas, replace native vegetation by exotic species and impermeable surfaces (i.e., buildings), resulting in local microclimate change and other aquatic and terrestrial physicochemical characteristics of riparian zones (McKinney 2002, Wen et al. 2017, Brasil et al. 2021). These effects convert natural environments into areas with homogeneous biotic and abiotic characteristics that also affect the diversity and composition of odonates (Calvão et al. 2018, Renner et al. 2018, Borges et al. 2021, Sganzerla et al. 2021). In anthropic areas, sensitive species (e.g., some Zygoptera) are substituted by generalist groups with tolerant traits (e.g., multivoltine, r-strategist and heliothermic), often simplifying odonate communities (De Marco et al. 2015, Dalzochio et al. 2018b, Oliveira-Júnior \& Juen 2019). Considering the vulnerability of many odonates species to environmental degradation, more inventories are needed to fill gaps about the diversity in these phytophysiognomies, even in anthropized areas.

Minas Gerais state present a great area composed by riparian forests and palm swamps, which are constantly decreasing with the intensification of urbanization and agriculture (Ratter et al. 1997, Velazco et al. 2019). The state has currently over 304 dragonfly species inventoried (Vilela 2020), with more than 20 new records and new species recently reported (Ávila Júnior et al. 2020, Vilela et al. 2020a, Vilela et al. 2020b). Mostly of these new data were obtained in poorly explored areas, such as Uberlândia, in Triângulo Mineiro region, western of the Minas Gerais state. Three inventories in this municipally found more than 60 species of adult dragonflies in just two palm swamp areas and in a degraded riparian forest (Vilela et al. 2016, Barbosa et al. 2019, Vilela et al. 2020a). Then, it is expected that a robust checklist can record a greater diversity of local dragonflies, since previous inventories explored few portions of the municipality territory. Moreover, some new species were also discovered in region, opening new opportunities for taxonomic studies. This is the case of Forcepsioneura machadorum Vilela, Venâncio and Santos, 2020, which was recently discovered, but species description was based only on males (Vilela et al. 2020b), remaining a taxonomic description of the female.

Thus, the main objective of this study is to conduct an inventory of dragonfly fauna in riparian forests and palm swamps of Uberlândia. As aquatic environments of the municipality are mainly represented by these types of vegetation, we will also indirectly perform a general inventory of the municipality. In addition, we also present some 
taxonomic notes on the females of $F$. machadorum. Our study aims to complement the state's Odonata record and distribution, contributing to reduce Linnean and Wallacean shortfalls.

\section{Material and Methods}

\section{Study area}

The territory of Uberlândia $\left(18^{\circ} 55^{\prime} 23^{\prime \prime} \mathrm{S}, 48^{\circ} 17^{\prime} 19^{\prime \prime} \mathrm{W}\right)$ is approximately $4,115 \mathrm{~km}^{2}$ (IBGE 2020), consisting of typical Cerrado biome vegetation and areas with agricultural (mainly livestock farming and monocultures) and urban activities. Local remnants of riparian vegetation consist of riparian forests, palm swamps and humid fields are located in areas with some type of protection (Cardoso \& Schiavini 2002, Maywald \& Marçal-Júnior 2013). The hydrography is represented by sub-basins and microbasins of the Paranaíba River, such as the Araguari and Uberabinha basins (Rosa et al. 2019). Part of this water is used for urban supply and agricultural activities in the municipality, which causes the pollution of some courses (Netto et al. 2011). Climate of region is AW type, according to the Köppen classification, characterized by hot and rainy summers (October-March) and dry and cold winters (April-September) (Alvares et al. 2013). The local mean annual temperature and rainfall are approximately $22^{\circ} \mathrm{C}$ and 1,500 $\mathrm{mm}$, respectively.

\section{Sampling}

We performed the samplings in 111 water bodies in the municipality (Figure 1). These localities consisted in lentic (ponds and lakes; $\mathrm{n}=$ 50) and lotic habitats up to third order (springs, streams and rivers; $\mathrm{n}=$ $61)$, located in or next to palm swamps $(n=54)$ and riparian forests $(n$ $=56$ ) in urban and rural areas (Figure 2; Table1). Site 57 was located in a highly urbanized area and the nearest riparian vegetation was more than $1.5 \mathrm{Km}$ away from this point. Therefore, we did not classify this site with a phytophysiognomy type and we excluded it from the phytophysiognomy analyses (see below). The urbanization class was determined according to urban limits of municipality. Two ponds (sites 3 and 57) and a small lotic section (site 89) were temporary and the others were permanent.

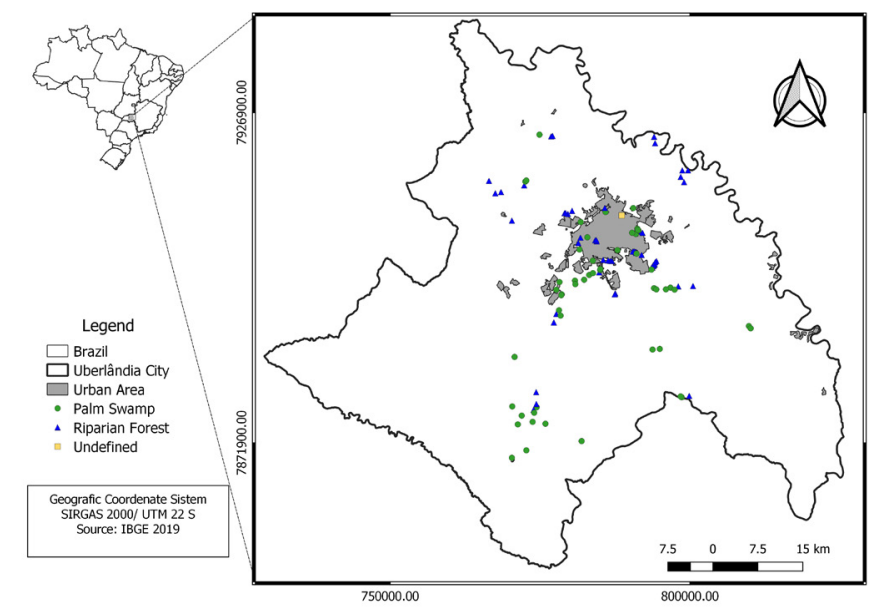

Figure 1. Sampled sites in Uberlândia, Minas Gerais, Brazil. Detailed information about the sites is presented in Table 1.
Collections in urban area were performed in parks with remnants of native vegetation and private lands. In rural area, seven localities were sampled in Conservation Units (sites 86-89, 90 and 92), and remaining areas in private farms with agricultural activities (fish farming, livestock and monoculture). Aquatic habitats and riparian vegetation in some degraded urban and rural areas have been intensively modified, losing their original characteristics. In some sites, there was a change in the type of habitat, usually due to damming or deviation of water from lotic courses to artificial ponds used for urban landscaping, animal drinking or fish tanks. The native vegetation in altered sites were partially or entirely replaced by exotic species (e.g., Brachiaria spp., Hyparrhenia spp. and Saccharum sp.). Despite these changes, we decided to maintain the original local phytophysiognomy classification, following other studies in the region (Borges et al. 2019, Barbosa et al. 2019).

We sampled most adult dragonflies with entomological nets, but light traps and larvae rearing were also used in some sites in order to sample species that are not so easily captured through traditional methods (Almeida et al. 2013, Pinto 2019). Active samplings were performed by one or two people during often one hour in a $100 \mathrm{~m}$ transect of each water body, from December 2018 to November 2020, during dry and rainy seasons. We sampled the dragonflies on hot, sunny days with few clouds between 9:00 am and 3:00 pm, conditions and period of greatest diversity and activity of the group (Calvão et al. 2018). Each site was sampled once, totaling an effort of approximately 110 hours. Collections with light trap (type "Luiz de Queiroz") were performed only at site 93 in October 2018. The trap was placed $3 \mathrm{~m}$ above the ground, with illumination effectuated by two black ultraviolet light that were activated for 12 hours (18:00 - 6:00 h) during seven days, totaling 84 sampling hours. We did not perform any other type of sampling method at this site. Finally, larvae specimens were sampled during two expeditions in August 2018 and May 2020 at site 10. The larvae were collected through D-network $(250 \mu \mathrm{m})$ scans close to emerged macrophytes. All F1-F0 larvae were reared in aquariums until adult emergence. Entomological nets were also used to sample adults at this site. Only larvae species not previously collected were reared.

Collected adults were stored in glassine envelopes (Cezário et al. 2020), kept in pure acetone during 12 hours and dried for 30 minutes under direct solar irradiation. The identification occurred at species level following the keys of Lencioni (2017), Garrison et al. (2006, 2010), Needham et al. (2000), and genus specific literature (e.g., revisions, synopses and species description). Collections were carried out under the authorization of ICMBio (SISBIO: 28398-1 and 28398-3) and IEF (064/2018). The material is deposited in the collection of the Laboratory of Ecology and Biodiversity of the Federal University of Sergipe.

\section{Statistical analysis}

Interpolation and extrapolation curves were performed for palm swamp and riparian forest, and from the combination of both to evaluate the effectiveness of odonate sampled for phytophysiognomies and municipality, respectively. Specimens of the site 57 were included only in overall municipality curve estimation due to absence of a defined phytophysiognomy. The curves (Hill number $q=0$ ) were based on specimen abundance, and confidence intervals (95\%) were determined from 1,000 bootstraps, with the extrapolation estimated to the triple of sampled specimens (Chao et al. 2014). The rarefaction curves were generated using the iNEXT (Hsieh et al. 2020) in software R version 
Venâncio, H. et al.
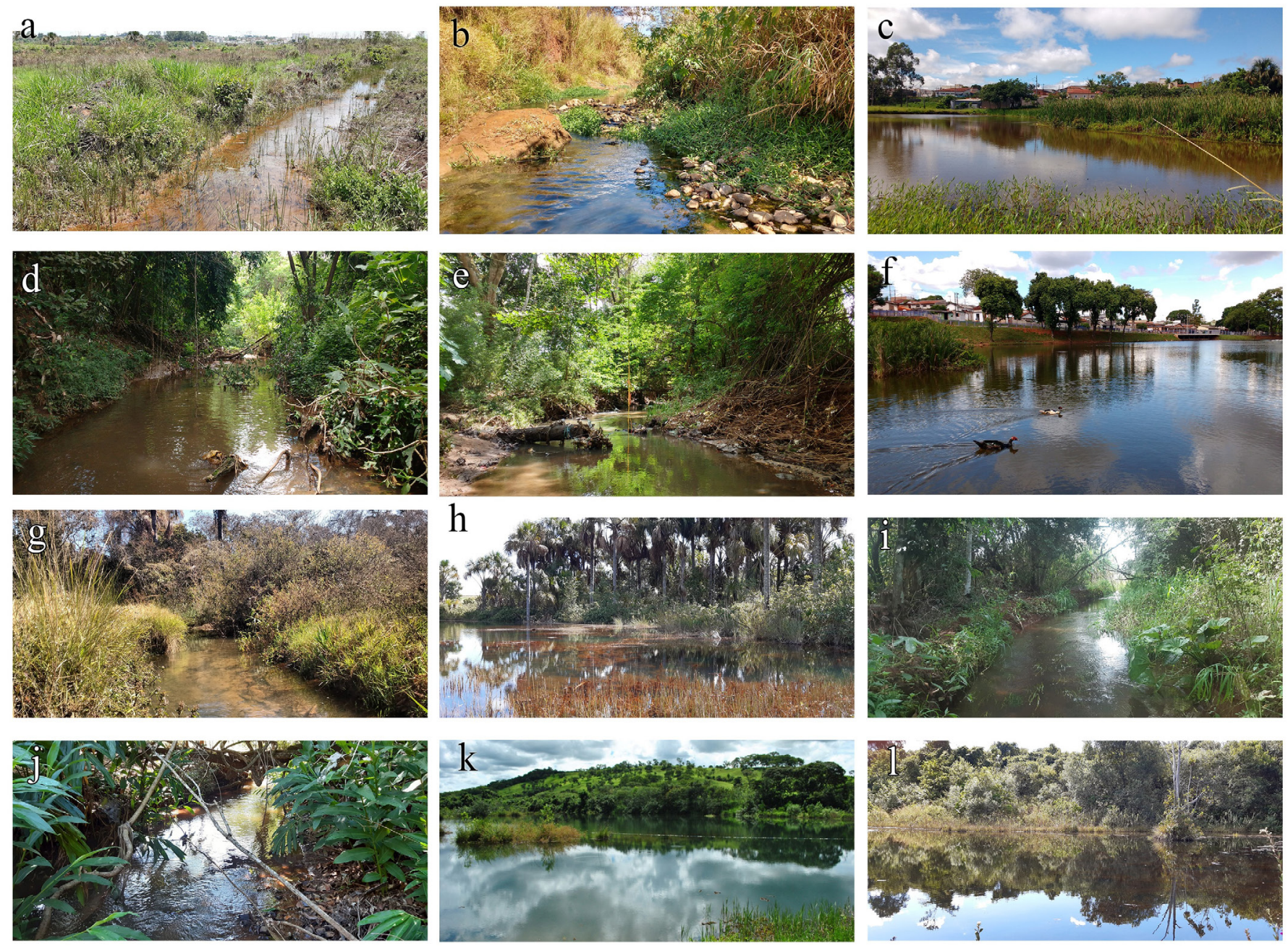

Figure 2. Environment characteristics of some sampled sites in Uberlândia, Minas Gerais, Brazil: (a) urban lotic palm swamp (site 53); (b) urban lotic palm swamp (site 8); (c) urban lentic palm swamp (site 25); (d) riparian forest located in public urban green area (site 26); (e) urban riparian forest located at urban park (site 41);(f) lentic urban pond near riparian forest; (g) rural palm swamp stream (site 94); (h) preserved rural lentic palm swamp (site 66); (i) rural riparian forest stream (site 103); (j) stream in a rural gallery forest (site 99); (k) lentic section surrounded by riparian forest (site 92); (1) rural pond in riparian forest segment (site 80).

4.0.2 (R Core Team 2020). Image editions were performed in Gimp version 2 (The GIMP Development Team 2019).

\section{Taxonomic notes}

To perform the female description, lateral views of habitus were scanned with an Epson V600 Perfection at colored 1,200 dpi with 200\% magnification. Structure images were made from multiple photographs focused at different levels, using Canon EOS T5 digital camera coupled to Tecnival stereomicroscope. Free hand illustrations were made using trace paper and scanned with Epson V600 Perfection at black and white 1,200 dpi with $100 \%$ magnification. Morphological terminology follows Lencioni (1999) and Garrison et al. (2010). All measurements are in millimeters $(\mathrm{mm})$.

Abbreviations: AB: abdomen length; Fw: fore wings; Hw: hind wings; MP: media posterior vein; Px: postnodal crossvein; Pt: pterostigma; RP2: radius posterior, second branch; S1-10: abdominal segments; TL: total length.

\section{Results}

\section{Odonata diversity}

We collected 2,154 individuals belonging to seven families, 46 genera and 101 species in the municipality (Table 2). Of this total, Anisoptera and Zygoptera presented $61 \%$ and 50\%, respectively, of species richness. Among the families, Libellulidae was the most specious group, comprising more than half of the species $(51 \%)$, followed by Coenagrionidae (36\%), Aeshnidae (5\%), Gomphidae (4\%), Calopterygidae, Dicteriadidae and Lestidae (4\%). Libellulidae and Coenagrionidae also presented the two richest genera (Erythrodiplax: 12\% spp. and Telebasis: 8\% spp.), and abundant [Erythrodiplax: 556 ( 25\%); Argia: 424 ( 20\%)], while Dicteriadidae and Lestidae were the less rich families (1\% each).

In general, sampled sites presented a mean richness of 6.43 (SD: \pm 3.18) species, varying from 1 to 18 species per water body. Urban sites presented 77 (mean \pm SD: $6.74 \pm 3.39)$ and rural $81(6.98 \pm 2.92)$ of the richness of this study, with 20 species occurring exclusively in the 
Table 1. Detailed information of the sampled sites in Uberlândia, Minas Gerais, Brazil. Legends: Liv: Livestock farming; Urb: Urbanization; Mono: Monoculture; Pisc: Pisciculture; Preserv: Preserved.

\begin{tabular}{|c|c|c|c|c|c|c|c|c|}
\hline Site & Coordinates & $\begin{array}{l}\text { Sampling } \\
\text { date }\end{array}$ & $\begin{array}{l}\text { Sampling } \\
\text { method }\end{array}$ & Area & Habitat & Vegetation & $\begin{array}{c}\text { Environmental } \\
\text { change in the } \\
\text { channel }\end{array}$ & $\begin{array}{c}\text { Land Use } \\
\text { beyond } \\
\text { riparian zone } \\
\end{array}$ \\
\hline 1 & $18^{\circ} 58^{\prime} 11^{\prime \prime} \mathrm{S}-48^{\circ} 17^{\prime} 42^{\prime \prime} \mathrm{W}$ & Mar/2019 & $\mathrm{EN}$ & Urban & Lotic & $\mathrm{RF}$ & Yes & Liv/Urb \\
\hline 2 & $18^{\circ} 53^{\prime} 03^{\prime \prime} \mathrm{S}-48^{\circ} 20^{\prime} 48^{\prime \prime} \mathrm{W}$ & Mar/2020 & $\mathrm{EN}$ & Urban & Lentic & $\mathrm{RF}$ & Yes & Liv/Urb \\
\hline 3 & $18^{\circ} 52^{\prime} 53^{\prime \prime} \mathrm{S}-48^{\circ} 21^{\prime} 03^{\prime \prime} \mathrm{W}$ & Mar/2020 & $\mathrm{EN}$ & Urban & Lentic & RF & Yes & Liv/Urb \\
\hline 4 & $18^{\circ} 52^{\prime} 53^{\prime} \mathrm{S}-48^{\circ} 20^{\prime} 58^{\prime \prime} \mathrm{W}$ & Mar/2020 & $\mathrm{EN}$ & Urban & Lotic & $\mathrm{RF}$ & No & Liv/Urb \\
\hline 5 & $18^{\circ} 58^{\prime} 18^{\prime \prime S}-48^{\circ} 18^{\prime} 17^{\prime \prime} \mathrm{W}$ & Out/2019 & $\mathrm{EN}$ & Urban & Lentic & PS & Yes & $\mathrm{Liv} / \mathrm{Urb}$ \\
\hline 6 & $18^{\circ} 52^{\prime} 45^{\prime \prime} \mathrm{S}-48^{\circ} 17^{\prime} 08^{\prime \prime} \mathrm{W}$ & Mar/2019 & $\mathrm{EN}$ & Urban & Lotic & PS & Yes & Urb \\
\hline 7 & $18^{\circ} 55^{\prime} 03^{\prime \prime} \mathrm{S}-48^{\circ} 18^{\prime} 50^{\prime \prime} \mathrm{W}$ & Jul/2019 & $\mathrm{EN}$ & Urban & Lotic & PS & Yes & Urb \\
\hline 8 & $18^{\circ} 53^{\prime} 40^{\prime \prime} \mathrm{S}-48^{\circ} 19^{\prime} 31^{\prime \prime} \mathrm{W}$ & $\mathrm{Jul} / 2019$ & EN & Urban & Lotic & PS & Yes & Urb \\
\hline 9 & $18^{\circ} 56^{\prime} 34^{\prime \prime} \mathrm{S}-48^{\circ} 13^{\prime} 41^{\prime \prime} \mathrm{W}$ & Jul/2019 & $\mathrm{EN}$ & Urban & Lotic & PS & Yes & Liv/Urb \\
\hline 10 & $18^{\circ} 57^{\prime} 42^{\prime \prime} \mathrm{S}-48^{\circ} 12^{\prime} 38^{\prime \prime} \mathrm{W}$ & $\begin{array}{c}\text { May/2019 } \\
\text { Jul/2020 }\end{array}$ & $\begin{array}{l}\text { EN } \\
\text { DN }\end{array}$ & Urban & Lentic & $\mathrm{RF}$ & Yes & Pisc/Urb \\
\hline 11 & $18^{\circ} 57^{\prime} 32^{\prime} \mathrm{S}-48^{\circ} 12^{\prime} 34^{\prime \prime} \mathrm{W}$ & May/2019 & $\mathrm{EN}$ & Urban & Lotic & $\mathrm{RF}$ & Yes & $\mathrm{Pisc} / \mathrm{Urb}$ \\
\hline 12 & $18^{\circ} 57^{\prime} 26^{\prime \prime} \mathrm{S}-48^{\circ} 12^{\prime} 28^{\prime \prime} \mathrm{W}$ & May/2019 & $\mathrm{EN}$ & Urban & Lentic & $\mathrm{RF}$ & Yes & Liv/Urb \\
\hline 13 & $18^{\circ} 57^{\prime} 29^{\prime \prime} \mathrm{S}-48^{\circ} 12^{\prime} 29^{\prime \prime} \mathrm{W}$ & Out/2019 & $\mathrm{EN}$ & Urban & Lotic & $\mathrm{RF}$ & Yes & Liv/Urb \\
\hline 14 & $18^{\circ} 57^{\prime} 21^{\prime \prime} \mathrm{S}-48^{\circ} 12^{\prime} 21^{\prime \prime} \mathrm{W}$ & $\mathrm{Jul} / 2020$ & $\mathrm{EN}$ & Urban & Lotic & RF & Yes & $\mathrm{Liv} / \mathrm{Urb}$ \\
\hline 15 & $18^{\circ} 57^{\prime} 38^{\prime \prime} \mathrm{S}-48^{\circ} 12^{\prime} 40^{\prime \prime} \mathrm{W}$ & Dez/2019 & $\mathrm{EN}$ & Urban & Lotic & RF & No & $\mathrm{Liv} / \mathrm{Urb}$ \\
\hline 16 & $18^{\circ} 57^{\prime} 51^{\prime \prime S}-48^{\circ} 12^{\prime} 43^{\prime \prime} \mathrm{W}$ & Dez/2019 & $\mathrm{EN}$ & Urban & Lentic & PS & Yes & $\mathrm{Pisc} / \mathrm{Urb}$ \\
\hline 17 & $18^{\circ} 57^{\prime} 26^{\prime \prime} \mathrm{S}-48^{\circ} 12^{\prime} 33^{\prime \prime} \mathrm{W}$ & Nov/2019 & $\mathrm{EN}$ & Rural & Lotic & $\mathrm{RF}$ & No & Liv \\
\hline 18 & $18^{\circ} 57^{\prime} 08^{\prime \prime} \mathrm{S}-48^{\circ} 12^{\prime} 13^{\prime \prime} \mathrm{W}$ & Out/2019 & $\mathrm{EN}$ & Urban & Lotic & $\mathrm{RF}$ & No & Liv \\
\hline 19 & $18^{\circ} 57^{\prime} 13^{\prime \prime} \mathrm{S}-48^{\circ} 12^{\prime} 23^{\prime \prime} \mathrm{W}$ & Out/2019 & $\mathrm{EN}$ & Urban & Lotic & $\mathrm{RF}$ & No & Liv \\
\hline 20 & $18^{\circ} 59^{\prime} 08^{\prime \prime} \mathrm{S}-48^{\circ} 21^{\prime} 31^{\prime \prime} \mathrm{W}$ & $\mathrm{Mar} / 2019$ & $\mathrm{EN}$ & Urban & Lentic & PS & Yes & $\mathrm{Urb} / \mathrm{Liv}$ \\
\hline 21 & $18^{\circ} 58^{\prime} 02^{\prime} \mathrm{S}-48^{\circ} 17^{\prime} 36^{\prime \prime} \mathrm{W}$ & Dez/2019 & EN & Urban & Lentic & PS & Yes & Urb \\
\hline 22 & $18^{\circ} 57^{\prime} 54^{\prime} \mathrm{S}-48^{\circ} 17^{\prime} 35^{\prime \prime} \mathrm{W}$ & Dez/2019 & $\mathrm{EN}$ & Urban & Lentic & PS & Yes & Urb \\
\hline 23 & $18^{\circ} 52^{\prime} 22^{\prime} \mathrm{S}-48^{\circ} 14^{\prime} 32^{\prime \prime} \mathrm{W}$ & Jul/2019 & $\mathrm{EN}$ & Urban & Lotic & PS & Yes & Urb \\
\hline 24 & $18^{\circ} 56^{\prime} 11^{\prime \prime S}-48^{\circ} 19^{\prime} 35^{\prime \prime} \mathrm{W}$ & $\mathrm{Jul} / 2019$ & EN & Urban & Lentic & PS & Yes & Urb \\
\hline 25 & $18^{\circ} 57^{\prime} 09^{\prime} \mathrm{S}-48^{\circ} 18^{\prime} 18^{\prime \prime} \mathrm{W}$ & $\mathrm{Mar} / 2019$ & $\mathrm{EN}$ & Urban & Lentic & PS & Yes & Urb \\
\hline 26 & $18^{\circ} 55^{\prime} 37^{\prime \prime S}-48^{\circ} 19^{\prime} 44^{\prime \prime} \mathrm{W}$ & Mar/2019 & $\mathrm{EN}$ & Urban & Lotic & $\mathrm{RF}$ & Yes & Urb \\
\hline 27 & $18^{\circ} 57^{\prime} 10^{\prime \prime S}-48^{\circ} 16^{\prime} 32^{\prime \prime} \mathrm{W}$ & $\mathrm{Feb} / 2019$ & $\mathrm{EN}$ & Urban & Lentic & $\mathrm{RF}$ & Yes & Urb \\
\hline 28 & $18^{\circ} 57^{\prime} 09^{\prime} \mathrm{S}-48^{\circ} 16^{\prime} 35^{\prime \prime} \mathrm{W}$ & $\mathrm{Feb} / 2019$ & $\mathrm{EN}$ & Urban & Lotic & $\mathrm{RF}$ & Yes & Urb \\
\hline 29 & $18^{\circ} 57^{\prime} 09^{\prime \prime} \mathrm{S}-48^{\circ} 16^{\prime} 43^{\prime \prime} \mathrm{W}$ & $\mathrm{Feb} / 2019$ & $\mathrm{EN}$ & Urban & Lotic & $\mathrm{RF}$ & Yes & Urb \\
\hline 30 & $18^{\circ} 57^{\prime} 06^{\prime \prime} \mathrm{S}-48^{\circ} 16^{\prime} 51^{\prime \prime} \mathrm{W}$ & $\mathrm{Feb} / 2019$ & $\mathrm{EN}$ & Urban & Lotic & $\mathrm{RF}$ & Yes & Urb \\
\hline 31 & $18^{\circ} 55^{\prime} 03^{\prime \prime S}-48^{\circ} 19^{\prime} 32^{\prime \prime} \mathrm{W}$ & $\operatorname{Mar} / 2019$ & $\mathrm{EN}$ & Urban & Lentic & $\mathrm{RF}$ & Yes & Urb \\
\hline 32 & $18^{\circ} 56^{\prime} 10^{\prime \prime} \mathrm{S}-48^{\circ} 15^{\prime} 59^{\prime \prime} \mathrm{W}$ & $\mathrm{Mar} / 2019$ & $\mathrm{EN}$ & Urban & Lotic & PS & Yes & Urb \\
\hline 33 & $18^{\circ} 54^{\prime} 23^{\prime \prime} \mathrm{S}-48^{\circ} 13^{\prime} 56^{\prime \prime} \mathrm{W}$ & $\mathrm{Feb} / 2019$ & $\mathrm{EN}$ & Urban & Lentic & $\mathrm{RF}$ & Yes & Urb \\
\hline 34 & $18^{\circ} 54^{\prime} 29^{\prime \prime} \mathrm{S}-48^{\circ} 13^{\prime} 53^{\prime \prime} \mathrm{W}$ & $\mathrm{Feb} / 2019$ & $\mathrm{EN}$ & Urban & Lotic & $\mathrm{RF}$ & Yes & Urb \\
\hline 35 & $18^{\circ} 54^{\prime} 31^{\prime \prime} \mathrm{S}-48^{\circ} 13^{\prime} 41^{\prime \prime} \mathrm{W}$ & $\mathrm{Feb} / 2019$ & $\mathrm{EN}$ & Urban & Lotic & $\mathrm{RF}$ & No & Urb \\
\hline 36 & $18^{\circ} 54^{\prime} 13^{\prime \prime S}-48^{\circ} 14^{\prime} 07^{\prime \prime} \mathrm{W}$ & $\mathrm{Feb} / 2019$ & $\mathrm{EN}$ & Urban & Lentic & PS & Yes & Urb \\
\hline 37 & $18^{\circ} 54^{\prime} 40^{\prime \prime} \mathrm{S}-48^{\circ} 14^{\prime} 15^{\prime \prime} \mathrm{W}$ & $\mathrm{Feb} / 2019$ & $\mathrm{EN}$ & Urban & Lentic & PS & Yes & Urb \\
\hline 38 & $18^{\circ} 54^{\prime} 35^{\prime \prime} \mathrm{S}-48^{\circ} 14^{\prime} 37^{\prime \prime} \mathrm{W}$ & $\mathrm{Feb} / 2019$ & $\mathrm{EN}$ & Urban & Lentic & PS & Yes & Urb \\
\hline 39 & $18^{\circ} 54^{\prime} 23^{\prime \prime S}-48^{\circ} 14^{\prime} 02^{\prime \prime} \mathrm{W}$ & Jan/2019 & $\mathrm{EN}$ & Urban & Lentic & PS & Yes & Urb \\
\hline 40 & $18^{\circ} 54^{\prime} 19^{\prime \prime} \mathrm{S}-48^{\circ} 14^{\prime} 04^{\prime \prime} \mathrm{W}$ & $\mathrm{Feb} / 2019$ & $\mathrm{EN}$ & Urban & Lentic & PS & Yes & Urb \\
\hline 41 & $18^{\circ} 56^{\prime} 16^{\prime \prime} \mathrm{S}-48^{\circ} 14^{\prime} 19^{\prime \prime} \mathrm{W}$ & $\operatorname{Mar} / 2019$ & $\mathrm{EN}$ & Urban & Lotic & $\mathrm{RF}$ & Yes & Urb \\
\hline 42 & $18^{\circ} 56^{\prime} 17^{\prime \prime} \mathrm{S}-48^{\circ} 14^{\prime} 12^{\prime \prime} \mathrm{W}$ & $\mathrm{Mar} / 2019$ & $\mathrm{EN}$ & Urban & Lotic & $\mathrm{RF}$ & Yes & Urb \\
\hline 43 & $18^{\circ} 56^{\prime} 20^{\prime} \mathrm{S}-48^{\circ} 14^{\prime} 10^{\prime \prime} \mathrm{W}$ & Mar/2019 & $\mathrm{EN}$ & Urban & Lotic & $\mathrm{RF}$ & Yes & Urb \\
\hline 44 & $18^{\circ} 56^{\prime} 15^{\prime \prime} \mathrm{S}-48^{\circ} 14^{\prime} 24^{\prime \prime} \mathrm{W}$ & Mar/2019 & $\mathrm{EN}$ & Urban & Lotic & $\mathrm{RF}$ & Yes & Urb \\
\hline 45 & $18^{\circ} 56^{\prime} 23^{\prime \prime} \mathrm{S}-48^{\circ} 14^{\prime} 13^{\prime \prime} \mathrm{W}$ & $\mathrm{Mar} / 2019$ & $\mathrm{EN}$ & Urban & Lotic & $\mathrm{RF}$ & Yes & Urb \\
\hline 46 & $18^{\circ} 56^{\prime} 16^{\prime \prime} \mathrm{S}-48^{\circ} 14^{\prime} 31^{\prime \prime} \mathrm{W}$ & Mar/2019 & $\mathrm{EN}$ & Urban & Lotic & $\mathrm{RF}$ & Yes & Urb \\
\hline 47 & $18^{\circ} 56^{\prime} 26^{\prime \prime} \mathrm{S}-48^{\circ} 14^{\prime} 10^{\prime \prime} \mathrm{W}$ & Mar/2019 & $\mathrm{EN}$ & Urban & Lentic & PS & Yes & Urb \\
\hline
\end{tabular}


Venâncio, H. et al.

Continuation..

\begin{tabular}{|c|c|c|c|c|c|c|c|c|}
\hline 48 & $18^{\circ} 52^{\prime} 23^{\prime \prime S}-48^{\circ} 17^{\prime} 14^{\prime \prime} \mathrm{W}$ & $\operatorname{Jan} / 2019$ & $\mathrm{EN}$ & Urban & Lotic & RF & Yes & Urb \\
\hline 49 & $18^{\circ} 57^{\prime} 04^{\prime \prime} \mathrm{S}-48^{\circ} 17^{\prime} 19^{\prime \prime} \mathrm{W}$ & $\mathrm{Jul} / 2019$ & $\mathrm{EN}$ & Urban & Lentic & RF & Yes & Urb \\
\hline 50 & $18^{\circ} 55^{\prime} 24^{\prime \prime} \mathrm{S}-48^{\circ} 17^{\prime} 57^{\prime \prime} \mathrm{W}$ & $\mathrm{Feb} / 2019$ & $\mathrm{EN}$ & Urban & Lentic & RF & Yes & Urb \\
\hline 51 & $18^{\circ} 55^{\prime} 17^{\prime \prime} \mathrm{S}-48^{\circ} 17^{\prime} 59^{\prime \prime} \mathrm{W}$ & Feb/2019 & $\mathrm{EN}$ & Urban & Lotic & RF & Yes & Urb \\
\hline 52 & $18^{\circ} 55^{\prime} 18^{\prime \prime S}-48^{\circ} 18^{\prime} 09^{\prime \prime} \mathrm{W}$ & $\mathrm{Feb} / 2019$ & $\mathrm{EN}$ & Urban & Lentic & $\mathrm{RF}$ & Yes & Urb \\
\hline 53 & $18^{\circ} 58^{\prime} 28^{\prime \prime} \mathrm{S}-48^{\circ} 18^{\prime} 43^{\prime \prime} \mathrm{W}$ & Out/2019 & $\mathrm{EN}$ & Urban & Lotic & PS & Yes & Urb/Liv \\
\hline 54 & $19^{\circ} 00^{\prime} 15^{\prime \prime} \mathrm{S}-48^{\circ} 21^{\prime} 13^{\prime \prime} \mathrm{W}$ & $\operatorname{Jan} / 2019$ & $\mathrm{EN}$ & Urban & Lentic & PS & Yes & Liv \\
\hline 56 & $19^{\circ} 00^{\prime} 15^{\prime \prime} \mathrm{S}-48^{\circ} 21^{\prime} 09^{\prime \prime} \mathrm{W}$ & Feb/2019 & $\mathrm{EN}$ & Urban & Lotic & PS & No & Liv \\
\hline 57 & $18^{\circ} 53^{\prime} 02^{\prime \prime} \mathrm{S}-48^{\circ} 15^{\prime} 38^{\prime \prime} \mathrm{W}$ & Out/2019 & $\mathrm{EN}$ & Urban & Lentic & - & Yes & Urb \\
\hline 58 & $18^{\circ} 59^{\prime} 17^{\prime \prime} \mathrm{S}-48^{\circ} 08^{\prime} 45^{\prime \prime} \mathrm{W}$ & $\operatorname{Jan} / 2019$ & $\mathrm{EN}$ & Rural & Lentic & $\mathrm{RF}$ & Yes & Liv/Mono \\
\hline 59 & $19^{\circ} 00^{\prime} 02^{\prime \prime S}-48^{\circ} 16^{\prime} 07^{\prime \prime} \mathrm{W}$ & $\operatorname{Jan} / 2019$ & $\mathrm{EN}$ & Rural & Lotic & $\mathrm{RF}$ & Yes & Liv \\
\hline 60 & $19^{\circ} 00^{\prime} 08^{\prime \prime S}-48^{\circ} 16^{\prime} 10^{\prime \prime} \mathrm{W}$ & $\operatorname{Jan} / 2019$ & $\mathrm{EN}$ & Rural & Lotic & RF & Yes & Liv \\
\hline 65 & $19^{\circ} 04^{\prime} 59^{\prime \prime} \mathrm{S}-48^{\circ} 11^{\prime} 49^{\prime \prime} \mathrm{W}$ & Mar/2020 & $\mathrm{EN}$ & Rural & Lentic & PS & Yes & Liv/Mon \\
\hline 66 & $19^{\circ} 05^{\prime} 05^{\prime \prime} \mathrm{S}-48^{\circ} 12^{\prime} 30^{\prime \prime} \mathrm{W}$ & Mar/2020 & $\mathrm{EN}$ & Rural & Lentic & PS & No & Mon \\
\hline 67 & $19^{\circ} 02^{\prime} 02^{\prime \prime} \mathrm{S}-48^{\circ} 21^{\prime} 55^{\prime \prime} \mathrm{W}$ & Apr/2019 & $\mathrm{EN}$ & Rural & Lotic & PS & Yes & Liv \\
\hline 68 & $19^{\circ} 01^{\prime} 40^{\prime \prime} \mathrm{S}-48^{\circ} 21^{\prime} 26^{\prime \prime} \mathrm{W}$ & Apr/2019 & $\mathrm{EN}$ & Rural & Lentic & PS & Yes & Mon \\
\hline 69 & $19^{\circ} 02^{\prime} 08^{\prime \prime} \mathrm{S}-48^{\circ} 21^{\prime} 18^{\prime \prime} \mathrm{W}$ & Nov/2019 & $\mathrm{EN}$ & Rural & Lentic & PS & Yes & Liv \\
\hline 70 & $19^{\circ} 14^{\prime} 20^{\prime \prime} \mathrm{S}-48^{\circ} 24^{\prime} 23^{\prime \prime} \mathrm{W}$ & Mar/2020 & $\mathrm{EN}$ & Rural & Lentic & PS & Yes & Mon \\
\hline 71 & $18^{\circ} 58^{\prime} 39^{\prime \prime} \mathrm{S}-48^{\circ} 18^{\prime} 54^{\prime \prime} \mathrm{W}$ & Out/2019 & $\mathrm{EN}$ & Urban & Lentic & PS & Yes & Liv \\
\hline 72 & $19^{\circ} 11^{\prime} 44^{\prime \prime} \mathrm{S}-48^{\circ} 23^{\prime} 46^{\prime \prime} \mathrm{W}$ & Mar/2020 & $\mathrm{EN}$ & Rural & Lentic & PS & Yes & Mon \\
\hline 73 & $19^{\circ} 11^{\prime} 14^{\prime \prime S}-48^{\circ} 24^{\prime} 48^{\prime \prime} \mathrm{W}$ & Mar/2020 & $\mathrm{EN}$ & Rural & Lentic & PS & Yes & Mon \\
\hline 74 & $19^{\circ} 12^{\prime} 03^{\prime \prime S}-48^{\circ} 25^{\prime} 11^{\prime \prime} \mathrm{W}$ & $\mathrm{Mar} / 2020$ & $\mathrm{EN}$ & Rural & Lentic & PS & Yes & Mon \\
\hline 81 & $18^{\circ} 59^{\prime} 28^{\prime \prime} \mathrm{S}-48^{\circ} 10^{\prime} 53^{\prime \prime} \mathrm{W}$ & $\mathrm{Fev} / 2020$ & $\mathrm{EN}$ & Rural & Lotic & PS & Yes & Liv/Mono \\
\hline 82 & 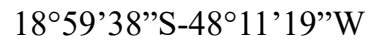 & Apr/2020 & $\mathrm{EN}$ & Rural & Lotic & PS & Yes & Liv \\
\hline 83 & $19^{\circ} 09^{\prime} 16^{\prime \prime} \mathrm{S}-48^{\circ} 09^{\prime} 45^{\prime \prime} \mathrm{W}$ & $\mathrm{Fev} / 2020$ & $\mathrm{EN}$ & Rural & Lentic & PS & Yes & Liv \\
\hline 84 & $19^{\circ} 09^{\prime} 23^{\prime} \mathrm{S}-48^{\circ} 09^{\prime} 35^{\prime \prime} \mathrm{W}$ & Fev/2020 & $\mathrm{EN}$ & Rural & Lotic & PS & Yes & Liv \\
\hline 85 & $19^{\circ} 09^{\prime} 11^{\prime \prime S}-48^{\circ} 08^{\prime} 58^{\prime \prime} \mathrm{W}$ & Fev/2020 & $\mathrm{EN}$ & Rural & Lotic & $\mathrm{RF}$ & Yes & Liv \\
\hline 86 & $19^{\circ} 10^{\prime} 57^{\prime \prime S}-48^{\circ} 23^{\prime} 39^{\prime \prime} \mathrm{W}$ & $\mathrm{Feb} / 2019$ & $\mathrm{EN}$ & Rural & Lentic & PS & No & Preserv \\
\hline 87 & $19^{\circ} 10^{\prime} 36^{\prime \prime} \mathrm{S}-48^{\circ} 23^{\prime} 40^{\prime \prime} \mathrm{W}$ & $\operatorname{Jan} / 2020$ & $\mathrm{EN}$ & Rural & Lotic & $\mathrm{RF}$ & No & Preserv \\
\hline 88 & $19^{\circ} 10^{\prime} 10^{\prime \prime S}-48^{\circ} 23^{\prime} 27^{\prime \prime} \mathrm{W}$ & $\mathrm{Jan} / 2020$ & $\mathrm{EN}$ & Rural & Lotic & $\mathrm{RF}$ & No & Preserv \\
\hline 89 & 1909'39”S-48 $23^{\circ} 13^{\prime \prime} \mathrm{W}$ & $\mathrm{Jan} / 2020$ & $\mathrm{EN}$ & Rural & Lotic & PS & Yes & Liv \\
\hline 90 & $18^{\circ} 49^{\prime} 30^{\prime \prime} \mathrm{S}-48^{\circ} 10^{\prime} 03^{\prime \prime} \mathrm{W}$ & Dez/2018 & $\mathrm{EN}$ & Rural & Lotic & $\mathrm{RF}$ & No & Preserv \\
\hline 91 & $18^{\circ} 49^{\prime} 56^{\prime \prime} \mathrm{S}-48^{\circ} 09^{\prime} 46^{\prime \prime} \mathrm{W}$ & Mar/2019 & $\mathrm{EN}$ & Rural & Lotic & $\mathrm{RF}$ & Yes & Liv \\
\hline 92 & $18^{\circ} 48^{\prime} 52^{\prime \prime} \mathrm{S}-48^{\circ} 09^{\prime} 24^{\prime \prime} \mathrm{W}$ & Apr/2019 & $\mathrm{EN}$ & Rural & Lentic & RF & Yes & Liv \\
\hline 93 & $18^{\circ} 49^{\prime} 04^{\prime \prime} \mathrm{S}-48^{\circ} 09^{\prime} 48^{\prime \prime} \mathrm{W}$ & Oct/2018 & LT & Rural & Lotic & $\mathrm{RF}$ & No & Preserv \\
\hline 94 & $19^{\circ} 02^{\prime} 39^{\prime \prime} \mathrm{S}-48^{\circ} 21^{\prime} 57^{\prime \prime} \mathrm{W}$ & Jul/2019 & $\mathrm{EN}$ & Rural & Lotic & PS & Yes & Liv \\
\hline 95 & $19^{\circ} 05^{\prime} 55^{\prime \prime} \mathrm{S}-48^{\circ} 25^{\prime} 37^{\prime \prime} \mathrm{W}$ & $\mathrm{Mar} / 2020$ & $\mathrm{EN}$ & Rural & Lotic & PS & Yes & Liv \\
\hline 96 & $18^{\circ} 45^{\prime} 50^{\prime \prime S}-48^{\circ} 23^{\prime} 29^{\prime \prime} \mathrm{W}$ & Mar/2020 & $\mathrm{EN}$ & Rural & Lotic & RF & Yes & Liv \\
\hline 97 & $19^{\circ} 15^{\prime} 04^{\prime \prime} \mathrm{S}-48^{\circ} 25^{\prime} 43^{\prime \prime} \mathrm{W}$ & Mar/2020 & $\mathrm{EN}$ & Rural & Lotic & PS & Yes & Mono \\
\hline 98 & $19^{\circ} 10^{\prime} 22^{\prime \prime} \mathrm{S}-48^{\circ} 25^{\prime} 40^{\prime \prime} \mathrm{W}$ & Mar/2020 & $\mathrm{EN}$ & Rural & Lotic & PS & Yes & Liv \\
\hline 99 & $18^{\circ} 53^{\prime} 39^{\prime \prime} \mathrm{S}-48^{\circ} 26^{\prime} 03^{\prime \prime} \mathrm{W}$ & Mar/2020 & $\mathrm{EN}$ & Rural & Lotic & RF & No & Liv \\
\hline 100 & $18^{\circ} 46^{\prime} 27^{\prime \prime} \mathrm{S}-48^{\circ} 12^{\prime} 33^{\prime \prime} \mathrm{W}$ & May/2020 & $\mathrm{EN}$ & Rural & Lotic & RF & Yes & Liv \\
\hline
\end{tabular}


Continuation.

\begin{tabular}{|c|c|c|c|c|c|c|c|c|}
\hline 101 & $18^{\circ} 50^{\prime} 06^{\prime} \mathrm{S}-48^{\circ} 28^{\prime} 16^{\prime \prime} \mathrm{W}$ & Apr/2020 & EN & Rural & Lotic & $\mathrm{RF}$ & Yes & Liv \\
\hline 102 & $18^{\circ} 51^{\prime} 07^{\prime \prime S}-48^{\circ} 27^{\prime} 09^{\prime \prime} \mathrm{W}$ & $\mathrm{Apr} / 2020$ & EN & Rural & Lentic & $\mathrm{RF}$ & Yes & Liv \\
\hline 103 & $18^{\circ} 51^{\prime} 12^{\prime \prime S}-48^{\circ} 27^{\prime} 40^{\prime \prime} \mathrm{W}$ & Apr/2020 & EN & Rural & Lotic & $\mathrm{RF}$ & Yes & Liv/Mono \\
\hline 104 & $18^{\circ} 59^{\prime} 34^{\prime \prime} \mathrm{S}-48^{\circ} 10^{\prime} 25^{\prime \prime} \mathrm{W}$ & Fev/2020 & EN & Rural & Lentic & PS & Yes & Liv \\
\hline 105 & $18^{\circ} 45^{\prime} 38^{\prime \prime S}-48^{\circ} 12^{\prime} 31^{\prime \prime} \mathrm{W}$ & May/2020 & EN & Rural & Lentic & $\mathrm{RF}$ & Yes & Pisc \\
\hline 106 & 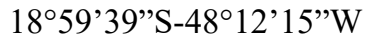 & Apr/2020 & EN & Rural & Lotic & PS & Yes & Liv/Mono \\
\hline 107 & $18^{\circ} 50^{\prime} 06^{\prime \prime} \mathrm{S}-48^{\circ} 24^{\prime} 49^{\prime \prime} \mathrm{W}$ & Mar/2020 & EN & Rural & Lentic & PS & Yes & Mon \\
\hline 108 & $18^{\circ} 50^{\prime} 01^{\prime \prime} \mathrm{S}-48^{\circ} 24^{\prime} 40^{\prime \prime} \mathrm{W}$ & Mar/2020 & EN & Rural & Lentic & PS & Yes & Mon \\
\hline 109 & $18^{\circ} 50^{\prime} 33^{\prime \prime} \mathrm{S}-48^{\circ} 24^{\prime} 55^{\prime \prime} \mathrm{W}$ & $\mathrm{Mar} / 2020$ & EN & Rural & Lotic & $\mathrm{RF}$ & No & Mon \\
\hline 110 & $1^{\circ} 02^{\prime} 50^{\prime \prime} \mathrm{S}-48^{\circ} 03^{\prime} 16^{\prime \prime} \mathrm{W}$ & $\mathrm{Feb} / 2019$ & EN & Rural & Lentic & PS & Yes & Liv \\
\hline 111 & $1^{\circ} 02^{\prime} 57^{\prime \prime} \mathrm{S}-48^{\circ} 03^{\prime} 17^{\prime \prime} \mathrm{W}$ & Feb/2019 & EN & Rural & Lentic & PS & No & Liv \\
\hline
\end{tabular}

first class, 24 in the second and 57 in both areas. Lentic habitats were richer than lotic (lentic: 79 species, $7.47 \pm 3.56$; lotic: 61 species, 6.35 \pm 2.77 ) and also presented more exclusively species (lentic: 40; lotic: 21; both: 39). Considering the phytophysiognomies, palm swamps and forests presented, respectively, a richness of $76(7.79 \pm 3.15)$ and 66 $(6.02 \pm 2.93)$ of the sampled species; and approximately 36 species were exclusive to palm swamps, 25 to forests and 41 were common to both habitats. Lestidae was the only family with all species common to all classes, while Dicteriadidae was specific to lotic forested sites.

Four specimens of four species (Aphylla distinguenda Campion, 1920, Phyllocycla cf. medusa Belle, 1988, Miathyria marcella Selys in Sagra, 1857, Tholymis citrina Hagen, 1867) were captured through light trap. One individual of Anax amazili Burmeister, 1839, several of Acanthagrion truncatum Selys, 1876 and three of Angelagrion nathaliae Lencioni, 2008 emerged in the laboratory. Some A. truncatum and all other species were sampled using entomological net.

Almost $50 \%$ of the sampled species are not included in any of the IUCN conservation categories; one species (Oligoclada xanthopleura Borror, 1931) was classified as Data Deficient (DD) and $45 \%$ were listed as (LC) least concern. However, Erythrodiplax ana GuillermoFerreira \& Vilela 2016 is considered endangered (EN).

The rarefaction and extrapolation curves suggested that samples were satisfactory, although the sampling of more specimens indicate a diversity increment in all cases (Figure 3). Considering the triple of estimated richness was approximately 93 for palm swamps, 72 for riparian forest and 126 for all habitats, we sampled more than $80 \%$ of the richness of dragonflies in each phytophysiognomy and in the municipality.

\section{New records for the State}

We found eight new species records for Minas Gerais: one Aeshnidae, one Gomphidae, three Libellulidae and three Coenagrionidae. Below, we present the distribution in the Brazilian states and a brief description of occurrence sites. Brazilian states are represented by respective acronyms: Acre (AC), Amapá (AP), Amazonas (AM), Bahia (BA), Espírito Santo (ES), Goiás (GO), Maranhão (MA), Mato Grosso (MT), Mato Grosso do Sul (MS), Pará (PA), Pernambuco (PE), Rio de Janeiro (RJ), Rio Grande do Sul (RS), Roraima (RO) and São Paulo (SP).

2.1 Neuraeschna claviforcipata Martin, $1909-10 \hat{0} ; 1$ q (Figure 4a). Distribution: AM (Koroiva et al., 2020). The two individuals were flying next to a spring of preserved riparian forest at site 35 (urban park). Due to low solar incidence in the course, both specimens had low flight speed during sampling.

2.2 Phyllocycla cf. medusa Belle, 1988 - 1 ㅇ (Figure 4b). Distribution: PA (Belle 1988). The specimen was collected in light trap at a riparian forest of the site 93, a conservation unit. The stream bed received direct sunlight and margins were completely shaded by tree vegetation.

2.3 Diastatops intensa Montgomery, 1940 - 4ðึ; 2 ㅇ (Figure 4c). Distribution: PA, AP, RS, MT, MS, SP, PE and RJ (FerreiraPeruquetti \& Fonseca-Gessner 2003, Silveira 2003, Costa et al. 2004, Garrison et al. 2006, Heckman 2006, Calvão et al. 2014, Dalzochio et al. 2018a, Rodrigues \& Roque 2017). All specimens were collected at site 92 , in a lentic stretch of a riparian forest of a conservation unit. Despite the predominance of grasses in the area, there was a preserved forest nearby and some macrophytes near the sampled transect, where specimens often were perching.

2.4. Oligoclada pachystigma Karsch, 1890 - $3 \widehat{\jmath}$ (Figure 4d). Distribution: AM, MS, MT, PA, SP (Costa et al. 2000, Pinto \& Lamas 2011, Carvalho et al. 2013, Rodrigues \& Roque 2017, Koroiva et al. 2020). All males were collected during mid-flight. The first specimen was found in the edge of a closed canopy palm swamp (site 62) and the other in a small pond in the same phytophysiognomy (site 65). Both areas were small fragments surrounded by livestock farming and corn crops (Zea mays L.).

2.5. Oligoclada xanthopleura Borror, 1931 - $1 \delta^{\Uparrow}$ (Figure 4e). Distribution: AM, PA, MT, MA (Pinto \& Lamas 2011, Carvalho et al. 2013, Calvão et al. 2014, Côrrea et al. 2014, Veras 2017, Koroiva et al. 2020). The specimen was collected in a palm swamp (site 5) with margins predominantly composed by Brachiaria sp. and exotic herbaceous species. Although the site was inserted in urban area, the nearest impervious structure was $\sim 500 \mathrm{~m}$ from the pond.

2.6. Angelagrion nathaliae Lencioni, $2008-2 \hat{0}$; 1 우 (Figure 4f). Distribution: SP (Lencioni 2008). The immature individuals were collected on Eichhornia crassipes Mart. in fish farming tanks near an urban gallery forest (site 10). The adults were reared during approximately one month until emergence at the laboratory. The larva and the male of this species are currently being described by Frederico Lencioni (pers. comm.).

2.7. Telebasis sanguinalis Calvert, $1909-1 \widehat{\partial}$ (Figure 4g). Distribution: MT, MS, AM, MA (Pinto \& Carvalho 2012, Koroiva et al. 2017, Rodrigues \& Roque 2017, Veras 2017, Koroiva et al. 2020). An individual was collected at a small palm swamp at 86, a different conservation unit from previous new records. The adjacent vegetation was composed of grasses and shrubs native to the Cerrado, near some M. flexuosa L. 
Venâncio, H. et al.

Table 2. List of dragonfly species sampled in Uberlândia, Minas Gerais, Brazil, indicating aquatic habitat type, vegetation type, area type, sampled season, abundance $(\mathrm{N})$ and occurrence sites. Le: Lentic; Lo: Lotic; R: Riparian Forest; P: Palm Swamp; Ru: Rural, U: Urban, D: Dry, R: Rainy. IUCN Red List Status: EN: Endangered, LC: Least Concern, DD: Data Deficient, "-“: Not categorized in the UCN list. New records for Minas Gerais are marked with an asterisk (*); "r" indicate larvae reared up to adults; 1 indicate species collected by light trap. Conservation status of the species were extracted from IUCN database (IUCN 2020).

\begin{tabular}{|c|c|c|c|c|c|c|c|}
\hline Taxon & Habitat & Vegetation & Area & Season & $\mathbf{N}$ & IUCN Status & Collection sites \\
\hline \multicolumn{8}{|l|}{ Anisoptera } \\
\hline \multicolumn{8}{|l|}{ Aeshnidae } \\
\hline Anax amazili (Burmeister, 1839) ${ }^{\mathrm{r}}$ & Le & $\mathrm{R}$ & $\mathrm{U}$ & $\mathrm{D}$ & 1 & $\mathrm{LC}$ & 10 \\
\hline Anax concolor Brauer 1865 & Le & $\mathrm{P}$ & $\mathrm{Ru}$ & $\mathrm{R}$ & 1 & $\mathrm{LC}$ & 86 \\
\hline Gynacantha nervosa Rambur, 1842 & Le & - & $\mathrm{U}$ & $\mathrm{R}$ & 1 & $\mathrm{LC}$ & 57 \\
\hline Coryphaeschna adnexa (Hagen, 1861) & Lo & $\mathrm{R}$ & $\mathrm{U}$ & $\mathrm{R}$ & 1 & $\mathrm{LC}$ & 35 \\
\hline Neuraeschna claviforcipata Martin. 1909* & Lo & $\mathrm{R}$ & $\mathrm{U}$ & $\mathrm{R}$ & 2 & - & 35 \\
\hline \multicolumn{8}{|l|}{ Gomphidae } \\
\hline Aphyla distinguenda (Campion, 1920) ${ }^{1}$ & Lo & $\mathrm{R}$ & $\mathrm{Ru}$ & $\mathrm{R}$ & 1 & - & 93 \\
\hline Gomphoides perdita (Förster, 1914) & Le & $\mathrm{P}$ & $\mathrm{Ru}$ & $\mathrm{R}$ & 3 & - & 73,104 \\
\hline Phyllocycla cf. medusa Belle, 1988*1 & Lo & $\mathrm{R}$ & $\mathrm{Ru}$ & $\mathrm{R}$ & 1 & - & 93 \\
\hline $\begin{array}{l}\text { Progomphus intricatus Hagen in Selys, } \\
1858\end{array}$ & Le, Lo & $\mathrm{P}, \mathrm{R}$ & $\mathrm{Ru}, \mathrm{U}$ & $\mathrm{D}, \mathrm{R}$ & 7 & $\mathrm{LC}$ & $30,67,68,85,106$ \\
\hline \multicolumn{8}{|l|}{ Libellulidae } \\
\hline Brachymesia herbida (Gundlach, 1889) & Le, Lo & $\mathrm{P}, \mathrm{R}$ & $\mathrm{Ru}, \mathrm{U}$ & $\mathrm{R}$ & 2 & $\mathrm{LC}$ & 5,88 \\
\hline Dasythemis venosa (Burmeister, 1839) & Lo & $\mathrm{P}$ & $\mathrm{Ru}$ & $\mathrm{D}, \mathrm{R}$ & 2 & - & 89,106 \\
\hline Diastatops intensa Montgomery, 1940* & Le & $\mathrm{R}$ & $\mathrm{Ru}$ & $\mathrm{D}$ & 6 & - & 92 \\
\hline Diastatops obscura (Fabricius, 1775) & $\mathrm{Le}$ & $\mathrm{R}$ & $\mathrm{Ru}$ & $\mathrm{D}$ & 1 & - & 102 \\
\hline Elasmothemis cannacrioides Calvert, 1906 & Le, Lo & $\mathrm{R}$ & $\mathrm{Ru}, \mathrm{U}$ & $\mathrm{D}, \mathrm{R}$ & 11 & - & $15,26,41,62,90,111$ \\
\hline Elasmothemis constricta (Calvert, 1898) & Le & $\mathrm{P}$ & $\mathrm{Ru}$ & $\mathrm{R}$ & 1 & - & 5 \\
\hline Erythemis credula (Hagen, 1861) & Le & $\mathrm{P}$ & $\mathrm{Ru}, \mathrm{U}$ & $\mathrm{R}$ & 4 & - & $16,73,86$ \\
\hline Erythemis peruviana (Rambur, 1842) & Le & $\mathrm{P}$ & $\mathrm{Ru}$ & $\mathrm{R}$ & 7 & $\mathrm{LC}$ & 25 \\
\hline Erythemis vesiculosa (Fabricius, 1775) & Le & $\mathrm{P}$ & $\mathrm{Ru}$ & $\mathrm{R}$ & 2 & $\mathrm{LC}$ & 21 \\
\hline Erythrodiplax ana Guillermo-Ferreira \& Vilela 2016 & Le, Lo & $\mathrm{P}$ & $\mathrm{Ru}, \mathrm{U}$ & $\mathrm{D}, \mathrm{R}$ & 3 & EN & $37,40,94$ \\
\hline Erythrodiplax castanea (Burmeister, 1839) & Le, Lo & $\mathrm{P}, \mathrm{R}$ & $\mathrm{Ru}, \mathrm{U}$ & $\mathrm{D}, \mathrm{R}$ & 79 & - & $\begin{array}{l}6-8,14,22,23,36-38,49,54,60,61, \\
63,68,73,74,82,84,92,94,101, \\
102,105,108,110\end{array}$ \\
\hline Erythrodiplax fusca (Rambur, 1842) & Le, Lo & $\mathrm{P}, \mathrm{R}$ & $\mathrm{Ru}, \mathrm{U}$ & $\mathrm{D}, \mathrm{R}$ & 193 & $\mathrm{LC}$ & $\begin{array}{l}2,3,5,8,10-12,14,16,20,23-25,28, \\
29,31-33,42,43,46,47,49,50,52, \\
55,56,61,62,64,65,67,68,70,72, \\
73,75,77,78,80-82,89,92,94,100, \\
101,104,106,107,108,109\end{array}$ \\
\hline Erythrodiplax juliana Ris, 1911 & Le, Lo & $\mathrm{P}, \mathrm{R}$ & $\mathrm{Ru}, \mathrm{U}$ & $\mathrm{D}, \mathrm{R}$ & 43 & $\mathrm{LC}$ & $\begin{array}{l}9,36,40,49,66,68,69,74,78,80- \\
82,85,86,100,105\end{array}$ \\
\hline Erythrodiplax lygaea Ris, 1911 & Le & $\mathrm{P}$ & $\mathrm{U}$ & $\mathrm{R}$ & 1 & $\mathrm{LC}$ & 71 \\
\hline Erythrodiplax latimaculata Ris, 1911 & Le, Lo & $\mathrm{P}, \mathrm{R}$ & $\mathrm{Ru}, \mathrm{U}$ & $\mathrm{D}, \mathrm{R}$ & 62 & & $\begin{array}{l}5,11,19,21,22,25,40,53,54,61,65 \\
66,69,70-73,75,78,80,83,85,86, \\
102,104,107,110,111\end{array}$ \\
\hline Erythrodiplax maculosa (Hagen, 1861) & $\mathrm{Le}$ & $\mathrm{P}$ & $\mathrm{Ru}, \mathrm{U}$ & $\mathrm{R}$ & 8 & $\mathrm{LC}$ & $40,70,71,78$ \\
\hline Erythrodiplax media Borror, 1942 & Le, Lo & $\mathrm{P}, \mathrm{R}$ & $\mathrm{Ru}, \mathrm{U}$ & $\mathrm{D}, \mathrm{R}$ & 104 & - & $\begin{array}{l}5,8,10,21,22,24,25,27,29,31- \\
33,36-38,40,45-47,49-52,65,66, \\
72,77,78,83,86,92,94,101,102, \\
104,111\end{array}$ \\
\hline Erythrodiplax ochracea (Burmeister, 1839) & $\mathrm{Le}$ & $\mathrm{R}$ & $\mathrm{Ru}$ & $\mathrm{R}$ & 2 & $\mathrm{LC}$ & 58 \\
\hline Erythrodiplax paraguayensis (Förster, 1904) & Le, Lo & $\mathrm{P}, \mathrm{R}$ & $\mathrm{Ru}, \mathrm{U}$ & $\mathrm{D}, \mathrm{R}$ & 34 & $\mathrm{LC}$ & $\begin{array}{l}5,9,10,16,21,22,31,32,40,49, \\
53,65,66,68,70,76,77,104,111\end{array}$ \\
\hline Erythrodiplax umbrata (Linnaeus, 1758) & Le, Lo & $\mathrm{P}, \mathrm{R}$ & $\mathrm{Ru}, \mathrm{U}$ & $\mathrm{D}, \mathrm{R}$ & 21 & $\mathrm{LC}$ & $6,3,10,21,31,40,77,78,54,111$ \\
\hline Erythrodiplax venusta (Kirby, 1897) & $\mathrm{Le}$ & $\mathrm{P}$ & $\mathrm{Ru}, \mathrm{U}$ & $\mathrm{R}$ & 6 & - & 21,110 \\
\hline Gynothemis venipunctata Calvert. 1909 & Le, Lo & $\mathrm{P}$ & $\mathrm{Ru}, \mathrm{U}$ & $\mathrm{D}, \mathrm{R}$ & 7 & - & $5,53,82,106$ \\
\hline Idiataphe amazonica (Kirby, 1889) & Le & $\mathrm{P}$ & $\mathrm{Ru}$ & $\mathrm{D}, \mathrm{R}$ & 3 & - & 73,76 \\
\hline
\end{tabular}

Continue... 
Dragonflies in a region of the Triângulo Mineiro

Continuation..

\begin{tabular}{|c|c|c|c|c|c|c|c|}
\hline Idiataphe longipes (Hagen, 1861) & Le & $P$ & $\mathrm{Ru}, \mathrm{U}$ & $\mathrm{R}$ & 2 & - & 65,71 \\
\hline Macrothemis heteronycha (Calvert, 1909) & Lo & $\mathrm{P}$ & $\mathrm{Ru}$ & $\mathrm{D}, \mathrm{R}$ & 4 & $\mathrm{LC}$ & $53,85,106$ \\
\hline Macrothemis imitans Karsch, 1890 & Le, Lo & $\mathrm{P}, \mathrm{R}$ & $\mathrm{Ru}, \mathrm{U}$ & $\mathrm{D}, \mathrm{R}$ & 6 & $\mathrm{LC}$ & $9,61,88,98$ \\
\hline Miathyria marcella (Selys in Sagra, 1857) ${ }^{1}$ & Lo & $\mathrm{R}$ & $\mathrm{Ru}$ & $\mathrm{R}$ & 1 & $\mathrm{LC}$ & 93 \\
\hline Miathyria simplex (Rambur, 1842) & Le & $\mathrm{R}$ & $\mathrm{Ru}$ & $\mathrm{D}$ & 1 & - & 92 \\
\hline Micrathyria catenata Calvert. 1909 & Le & $\mathrm{P}$ & $\mathrm{Ru}, \mathrm{U}$ & $\mathrm{R}$ & 11 & $\mathrm{LC}$ & $16,25,72,74$ \\
\hline Micrathyria cf. hypodidyma Calvert, 1906 & Lo & $\mathrm{R}$ & $\mathrm{Ru}$ & $\mathrm{R}$ & 1 & - & 87 \\
\hline Micrathyria hesperis Ris, 1911 & Le & $\mathrm{P}$ & $\mathrm{Ru}, \mathrm{U}$ & $\mathrm{R}$ & 9 & - & $25,27,47,52,72,75$ \\
\hline Micrathyria ocellata Martin, 1897 & Le & $P$ & $\mathrm{U}$ & $\mathrm{R}$ & 9 & - & $21,25,50,54$ \\
\hline Micrathyria pirassunungae Santos, 1953 & Le & $P$ & $\mathrm{Ru}, \mathrm{U}$ & $\mathrm{R}$ & 7 & - & $20,55,66,73$ \\
\hline Micrathyria pseudeximia Westfall, 1992 & Le, Lo & $\mathrm{P}, \mathrm{R}$ & $\mathrm{Ru}, \mathrm{U}$ & $\mathrm{D}, \mathrm{R}$ & 20 & - & $\begin{array}{l}16,25,29,45,46,52,69,71,73,86 \\
87,102\end{array}$ \\
\hline Micrathyria spuria (Selys, 1900) & Le & $\mathrm{P}$ & $\mathrm{Ru}, \mathrm{U}$ & $\mathrm{D}, \mathrm{R}$ & 6 & $\mathrm{LC}$ & $5,102,110$ \\
\hline Nephepeltia berlai Santos, 1950 & Le & $\mathrm{P}$ & $\mathrm{U}$ & $\mathrm{R}$ & 3 & - & $5,21,22$ \\
\hline Oligoclada laetitia Ris, 1911 & Le & $\mathrm{P}$ & $\mathrm{Ru}, \mathrm{U}$ & $\mathrm{R}$ & 4 & $\mathrm{LC}$ & 40,110 \\
\hline Oligoclada pachystigma Karsch, 1890* & Le, Lo & $P$ & $\mathrm{Ru}$ & $\mathrm{D}, \mathrm{R}$ & 3 & - & 62,65 \\
\hline Oligoclada xanthopleura Borror, 1931* & Le & $\mathrm{P}$ & $\mathrm{U}$ & $\mathrm{R}$ & 1 & DD & 5 \\
\hline Orthemis aequilibris Calvert, 1909 & Le, Lo & $\mathrm{P}$ & $\mathrm{Ru}, \mathrm{U}$ & $\mathrm{D}, \mathrm{R}$ & 3 & - & $24,32,104$ \\
\hline Orthemis cultriformis Calvert, 1899 & Lo & $\mathrm{R}$ & $\mathrm{Ru}, \mathrm{U}$ & $\mathrm{R}$ & 3 & - & 43,87 \\
\hline Orthemis discolor (Burmeister, 1839) & Le, Lo & $\mathrm{P}, \mathrm{R}$ & $\mathrm{Ru}, \mathrm{U}$ & $\mathrm{D}, \mathrm{R}$ & 18 & $\mathrm{LC}$ & $\begin{array}{l}2,10,19,23,46-48,55,69,73,85 \\
102,108\end{array}$ \\
\hline Pantala flavescens (Fabricius, 1798) & Le, Lo & $\mathrm{P}, \mathrm{R}$ & $\mathrm{Ru}, \mathrm{U}$ & $\mathrm{D}, \mathrm{R}$ & 6 & & $7,10,26,67,68,91$ \\
\hline Perithemis icteroptera (Selys in Sagra, 1857) & Le & $P$ & $\mathrm{U}$ & $\mathrm{R}$ & 6 & $\mathrm{LC}$ & 47 \\
\hline Perithemis lais (Perty, 1834) & Le & $\mathrm{P}, \mathrm{R}$ & $\mathrm{Ru}, \mathrm{U}$ & $\mathrm{R}$ & 14 & $\mathrm{LC}$ & $22,39,52,54,55,75$ \\
\hline Perithemis tenera (Say, 1840) & Le, Lo & $\mathrm{P}, \mathrm{R}$ & $\mathrm{U}$ & $\mathrm{D}, \mathrm{R}$ & 13 & $\mathrm{LC}$ & $5,8,24,25,27,54,55$ \\
\hline Tauriphila argo (Hagen, 1869) & Le & $\mathrm{P}$ & $\mathrm{U}$ & $\mathrm{R}$ & 1 & $\mathrm{LC}$ & 5 \\
\hline Tholymis citrina Hagen, $1867^{1}$ & Lo & $\mathrm{R}$ & $\mathrm{Ru}$ & $\mathrm{R}$ & 1 & $\mathrm{LC}$ & 93 \\
\hline Tramea abdominalis (Rambur, 1842) & Le & $\mathrm{P}$ & $\mathrm{U}$ & $\mathrm{R}$ & 3 & $\mathrm{LC}$ & 16 \\
\hline Tramea binotata (Rambur, 1842) & Le & $\mathrm{P}$ & $\mathrm{Ru}, \mathrm{U}$ & $\mathrm{R}$ & 3 & $\mathrm{LC}$ & $39,72,110$ \\
\hline Uracis siemensi Kirby, 1897 & Lo & $P$ & $\mathrm{U}$ & $\mathrm{R}$ & 1 & - & 17 \\
\hline Zenithoptera lanei Santos, 1941 & Le, Lo & $\mathrm{P}$ & $\mathrm{Ru}, \mathrm{U}$ & $\mathrm{R}$ & 10 & - & $5,22,53,66,71$ \\
\hline \multicolumn{8}{|l|}{ Zygoptera } \\
\hline \multicolumn{8}{|l|}{ Calopterygidae } \\
\hline Hetaerina rosea Selys, 1853 & Le, Lo & $\mathrm{P}, \mathrm{R}$ & $\mathrm{Ru}, \mathrm{U}$ & $\mathrm{D}, \mathrm{R}$ & 90 & - & $\begin{array}{l}1,4,6,7,11,13,15,19,26,30,32, \\
41,42,45,46,51,58,67,88,90, \\
94-98,103,109\end{array}$ \\
\hline Mnesarete guttifera (Selys,1873) & Lo & $\mathrm{R}$ & $\mathrm{Ru}$ & $\mathrm{R}$ & 1 & $\mathrm{LC}$ & 90 \\
\hline \multicolumn{8}{|l|}{ Coenagrionidae } \\
\hline Acanthagrion aepiolum Tennessen, 2004 & Le, Lo & $\mathrm{P}, \mathrm{R}$ & $\mathrm{Ru}, \mathrm{U}$ & $\mathrm{D}, \mathrm{R}$ & 79 & $\mathrm{LC}$ & $\begin{array}{l}9,21,24,31,33,39,41,43,45,46, \\
54,55,58,59,64,75,103,105\end{array}$ \\
\hline Acanthagrion gracile (Rambur, 1842) & Le, Lo & $\mathrm{P}, \mathrm{R}$ & $\mathrm{Ru}, \mathrm{U}$ & $\mathrm{D}, \mathrm{R}$ & 110 & - & $\begin{array}{l}2,3,5-8,10-12,14,20-23,27-29,33 \\
38,39,47,49,52,54,55,59,61,64,70 \\
72,74,75,79,80,83,94,100,102,105\end{array}$ \\
\hline Acanthagrion lancea Selys, 1876 & Le & $\mathrm{R}$ & $\mathrm{U}$ & $\mathrm{R}$ & 2 & $\mathrm{LC}$ & 5,31 \\
\hline Acanthagrion minutum Leonard, 1977 & Le & $\mathrm{P}$ & $\mathrm{Ru}, \mathrm{U}$ & $\mathrm{D}, \mathrm{R}$ & 5 & $\mathrm{LC}$ & $5,73,77,102$ \\
\hline Acanthagrion temporale Selys, 1876 & Le & $\mathrm{P}, \mathrm{R}$ & $\mathrm{Ru}, \mathrm{U}$ & $\mathrm{D}, \mathrm{R}$ & 16 & - & $\begin{array}{l}5,10,16,24,65,69,71,72,80,102 \\
2,3,5,10,16,24,25,28,36,37,40\end{array}$ \\
\hline Acanthagrion truncatum Selys, $1876^{\mathrm{r}}$ & Le, Lo & $\mathrm{P}, \mathrm{R}$ & $\mathrm{Ru}, \mathrm{U}$ & $\mathrm{D}, \mathrm{R}$ & 201 & $\mathrm{LC}$ & $\begin{array}{l}47,49,53,54,60,61,65,69,71,72, \\
80,89,94,101,102,104,106,107, \\
108,110,111\end{array}$ \\
\hline Angelagrion nathaliae Lencioni, $2008^{\mathrm{r} *}$ & Le & $\mathrm{R}$ & $\mathrm{U}$ & $\mathrm{D}$ & 3 & - & 10 \\
\hline Argia lilacina Selys, 1865 & Le, Lo & $\mathrm{P}, \mathrm{R}$ & $\mathrm{Ru}, \mathrm{U}$ & $\mathrm{D}, \mathrm{R}$ & 82 & - & $\begin{array}{l}8,9,10,14,17,33,53,54,61,62,67 \\
68,78,81,83,84,85,89,100,109\end{array}$ \\
\hline Argia mollis Hagen in Selys, 1865 & Lo & $\mathrm{P}, \mathrm{R}$ & $\mathrm{Ru}, \mathrm{U}$ & $\mathrm{D}, \mathrm{R}$ & 56 & $\mathrm{LC}$ & $\begin{array}{l}6,17,18,35,41,42,44,46,56,60 \\
62,79,87,89,96,100\end{array}$ \\
\hline Argia reclusa Selys, 1865 & Lo & $\mathrm{P}, \mathrm{R}$ & $\mathrm{R}, \mathrm{U}$ & $\mathrm{D}, \mathrm{R}$ & 275 & & $\begin{array}{l}1,4,8,9,11,13,15,18,19,26-30,32,34, \\
35,41-46,56,59,60,64,83,89,90,91,94, \\
95,96-99,103,109\end{array}$ \\
\hline
\end{tabular}


Venâncio, H. et al.

Continuation...

\begin{tabular}{|c|c|c|c|c|c|c|c|}
\hline Argia tamoyo Calvert, 1909 & Lo & $\mathrm{R}$ & $\mathrm{Ru}, \mathrm{U}$ & $\mathrm{D}, \mathrm{R}$ & 8 & $\mathrm{LC}$ & $51,59,60,63$ \\
\hline Argia sp. & Lo & $\mathrm{R}$ & $\mathrm{U}$ & $\mathrm{R}$ & 3 & - & 17,18 \\
\hline Cyanallagma nigrinuchale (Selys, 1876) & Le, Lo & $\mathrm{R}$ & $\mathrm{Ru}$ & $\mathrm{D}, \mathrm{R}$ & 4 & - & 84,102 \\
\hline Epipleoneura venezuelensis Rácenis, 1955 & Le, Lo & $\mathrm{P}, \mathrm{R}$ & $\mathrm{Ru}, \mathrm{U}$ & $\mathrm{D}, \mathrm{R}$ & 27 & - & $\begin{array}{l}2,4,33,34,53,88,90,91,95 \\
98,103\end{array}$ \\
\hline Epipleoneura williamsoni Santos, 1957 & Le, Lo & $\mathrm{P}, \mathrm{R}$ & $\mathrm{Ru}, \mathrm{U}$ & $\mathrm{D}, \mathrm{R}$ & 20 & $\mathrm{LC}$ & $11,12,17,18,55,64,103$ \\
\hline $\begin{array}{l}\text { Forcepsioneura machadorum Vilela, } \\
\text { Venâncio \& Santos, } 2020\end{array}$ & Lo & $\mathrm{R}$ & $\mathrm{U}$ & $\mathrm{R}$ & 5 & - & $17-19$ \\
\hline Homeoura chelifera (Selys, 1876) & Le, Lo & $\mathrm{P}, \mathrm{R}$ & $\mathrm{Ru}, \mathrm{U}$ & $\mathrm{D}, \mathrm{R}$ & 20 & - & $17,24,36,58,68,104,110,111$ \\
\hline Homeoura lindneri (Ris, 1928) & $\mathrm{Le}$ & $\mathrm{P}, \mathrm{R}$ & $\mathrm{Ru}, \mathrm{U}$ & $\mathrm{D}, \mathrm{R}$ & 16 & - & $10,21,31,65,92,110$ \\
\hline Ischnura capreolus (Hagen, 1861) & Le, Lo & $\mathrm{P}, \mathrm{R}$ & $\mathrm{Ru}, \mathrm{U}$ & $\mathrm{D}, \mathrm{R}$ & 23 & - & $\begin{array}{l}8,10,49,71,72,77,83,92,101 \\
102,104,108\end{array}$ \\
\hline Ischnura fluviatilis Selys, 1876 & Le, Lo & $\mathrm{P}, \mathrm{R}$ & $\mathrm{Ru}, \mathrm{U}$ & $\mathrm{D}, \mathrm{R}$ & 10 & $\mathrm{LC}$ & $10,25,40,47,51,63,85$ \\
\hline Nehalennia minuta (Selys in Sagra, 1857) & $\mathrm{Le}$ & $\mathrm{P}$ & $\mathrm{Ru}$ & $\mathrm{R}$ & 1 & - & 86 \\
\hline Neoneura sylvatica Hagen in Selys, 1886 & Le, Lo & $\mathrm{P}, \mathrm{R}$ & $\mathrm{Ru}, \mathrm{U}$ & $\mathrm{D}, \mathrm{R}$ & 14 & - & $\begin{array}{l}4,11,12,30,64,67,92,95,101 \\
103,105\end{array}$ \\
\hline Oxyagrion basale Selys, 1876 & Lo & $\mathrm{R}$ & $\mathrm{U}$ & $\mathrm{R}$ & 8 & - & $29,42,43$ \\
\hline Oxyagrion chapadense Costa, 1978 & Le, Lo & $\mathrm{P}, \mathrm{R}$ & $\mathrm{Ru}, \mathrm{U}$ & $\mathrm{D}, \mathrm{R}$ & 56 & - & $\begin{array}{l}6,8,13,14,19,26-28,30,32,41, \\
42,48,51,64,79,87,89,98,103\end{array}$ \\
\hline Oxyagrion microstigma (Selys, 1876) & Le, Lo & $\mathrm{P}, \mathrm{R}$ & $\mathrm{Ru}$ & $\mathrm{D}, \mathrm{R}$ & 7 & - & $62,71,72,80,82$ \\
\hline Oxyagrion terminale Selys, 1876 & Le, Lo & $\mathrm{P}, \mathrm{R}$ & $\mathrm{Ru}, \mathrm{U}$ & $\mathrm{D}, \mathrm{R}$ & 31 & - & $\begin{array}{l}6,17,23,28,29,36,38,42,43 \\
51,79\end{array}$ \\
\hline Protoneura tenuis Selys, 1860 & Lo & $\mathrm{R}$ & $\mathrm{U}$ & $\mathrm{R}$ & 2 & $\mathrm{LC}$ & 18,19 \\
\hline Telebasis carmesina Calvert, 1909 & Le & $\mathrm{P}, \mathrm{R}$ & $\mathrm{Ru}, \mathrm{U}$ & $\mathrm{D}, \mathrm{R}$ & 51 & $\mathrm{LC}$ & $\begin{array}{l}10,16,36,37,66,69,70,72,74 \\
77,80,86,108\end{array}$ \\
\hline Telebasis coccinea (Selys, 1876) & Lo & $\mathrm{P}, \mathrm{R}$ & $\mathrm{Ru}, \mathrm{U}$ & $\mathrm{D}, \mathrm{R}$ & 31 & - & $\begin{array}{l}20,22,39,66,70,71,73,77,80 \\
86,102,104\end{array}$ \\
\hline Telebasis corallina (Selys, 1876) & Lo & $\mathrm{P}$ & $\mathrm{Ru}$ & $\mathrm{D}$ & 1 & $\mathrm{LC}$ & 80 \\
\hline Telebasis filiola (Perty, 1834) & Le, Lo & $\mathrm{P}, \mathrm{R}$ & $\mathrm{U}$ & $\mathrm{R}$ & 20 & - & $25,28,50$ \\
\hline Telebasis griffinii (Martin, 1896) & Le, Lo & $\mathrm{R}$ & $\mathrm{Ru}, \mathrm{U}$ & $\mathrm{D}, \mathrm{R}$ & 20 & $\mathrm{LC}$ & $38,51,92$ \\
\hline Telebasis sanguinalis Calvert, 1909* & Le & $\mathrm{P}$ & $\mathrm{Ru}$ & $\mathrm{R}$ & 1 & $\mathrm{LC}$ & 86 \\
\hline Telebasis simulacrum (Calvert, 1909)* & Le & $\mathrm{P}$ & $\mathrm{Ru}, \mathrm{U}$ & $\mathrm{R}$ & 8 & $\mathrm{LC}$ & 22,111 \\
\hline Telebasis willinki Fraser, 1948 & Le & $\mathrm{P}, \mathrm{R}$ & $\mathrm{Ru}, \mathrm{U}$ & $\mathrm{D}, \mathrm{R}$ & 9 & $\mathrm{LC}$ & $2,27,92$ \\
\hline Tigriagrion aurantinigrum Calvert, 1909 & Le, Lo & $\mathrm{P}, \mathrm{R}$ & $\mathrm{Ru}, \mathrm{U}$ & $\mathrm{D}, \mathrm{R}$ & 22 & - & $\begin{array}{l}7,11,12,17,22,58,64,75,87 \\
94,101\end{array}$ \\
\hline \multicolumn{8}{|l|}{ Dicteriadidae } \\
\hline Heliocharis amazona Selys, 1853 & Lo & $\mathrm{R}$ & $\mathrm{Ru}, \mathrm{U}$ & $\mathrm{R}$ & 10 & - & $15,88,90$ \\
\hline \multicolumn{8}{|l|}{ Lestidae } \\
\hline Lestes forficula Rambur, 1842 & Le, Lo & $\mathrm{P}, \mathrm{R}$ & $\mathrm{Ru}, \mathrm{U}$ & $\mathrm{R}$ & 15 & $\mathrm{LC}$ & $3,16,21,59,72,74,77,111$ \\
\hline
\end{tabular}

2.8. Telebasis simulacrum (Calvert, 1909) - 7ठ; 1 q (Figure 4h). Distribution: RO, MT, MS, (Garrison 2009, Juen \& De Marco 2012, Rodrigues \& Roque 2017). We recorded these specimens at a degraded urban palm swamp adjacent to some small forest fragments (site 22), and at a rural palm swamp with a predominance of native grasses (site 111). All specimens were perching in emerged macrophytes at the time of sampling.

\section{Taxonomy}

Few months after the publication of $F$. machadorum, we collected three females in the type locality, including a tandem pair. Thus, here we describe the female of $F$. machadorum (Figure 5).
Head (Figure 5a). Labium, mandible and genae pale colored; anteclypeus dark brown with a brown spot at the center; remainder of the head black, except for a longitudinal pale dorsal stripe covering along postocular spots and occipital bar areas; antennifer pale colored with a pale ring at the apex; antenna dark brown, except for pale coloration on the posterior surface of pedicel; rear of the head pale colored.

Thorax (Figures $5 \mathrm{a}-\mathrm{d}$ ). Anterior lobe of prothorax dark brown dorsally, pale laterally; medial lobe dark brown except for pale areas in the propleuron, with two lateral tubercles; hind lobe rectangular with a slightly concave medial depression, laterally blunt with posterolateral corners forming rounded angles. Pterothorax with a thick black/metallic green stripe dorsally, except for a brown stripe on the interpleural suture; metepisternum and metepimeron pale colored except for a darker stripe 


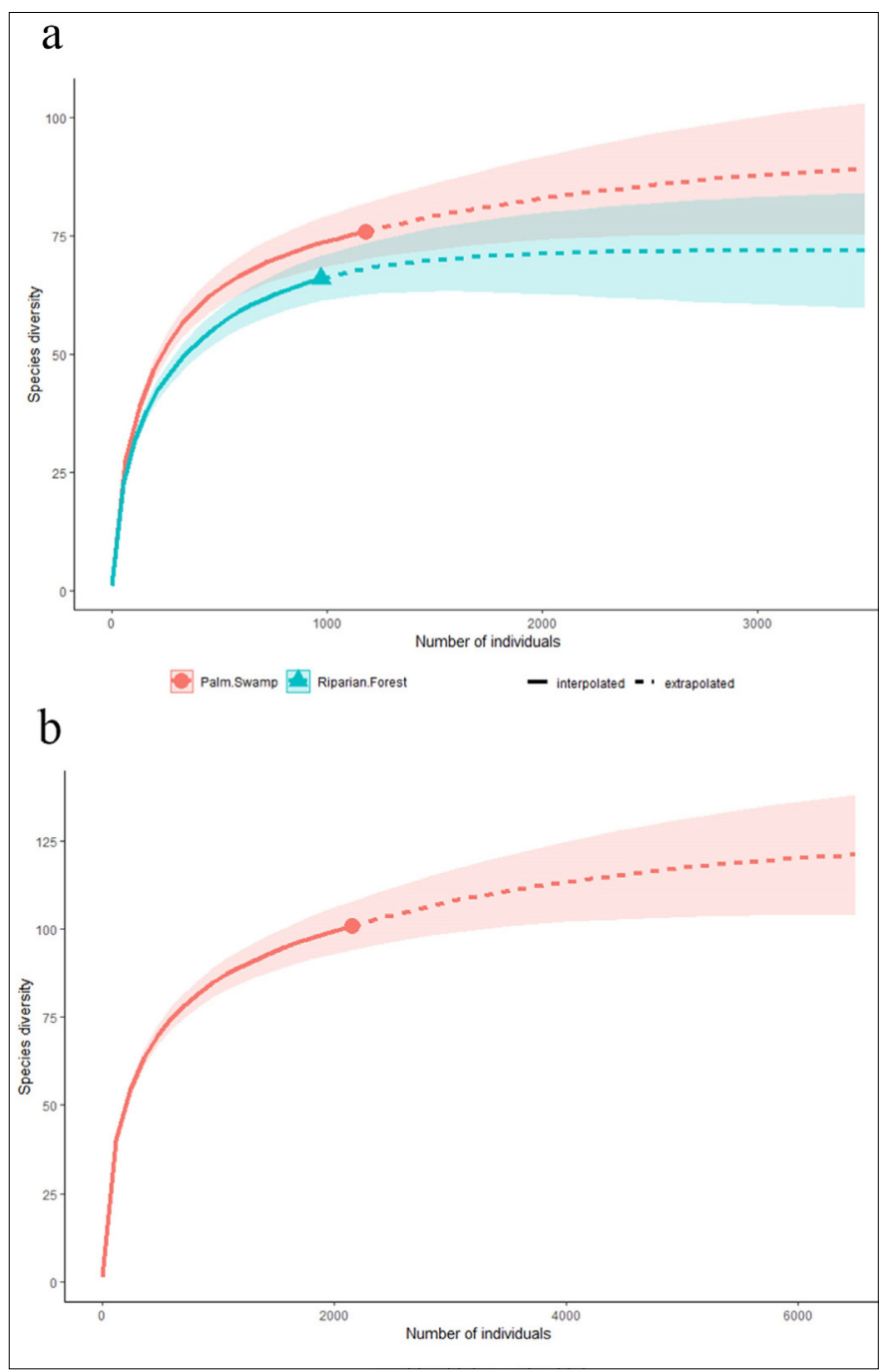

Figure 3. Accumulation curve (solid curve) and extrapolation (hatched curve) with $95 \%$ confidence interval (shaded area) of dragonflies sampled in (a) palm swamps and riparian forests and (b) general samples of Uberlândia, Minas Gerais, Brazil.

on the upper posterior portion of metepimeron and a small dark spot on the posterior metepisternum/metepimeron suture; venter pale.

Legs (Figure 5a). Overall coloration pale, femoral-tibial and tarsal articulations brown; spurs light brown.

Wings (Figure 5a). Hyaline; venation dark brown; Pt dark brown with pale contours; MP reaches anal margin 0.5 cell distal to the vein descending from subnodus; RP2 originating at $\mathrm{Px} 5$ in Fw, at $\mathrm{Px} 4$ in $\mathrm{Hw}$; $11 \mathrm{Px}$ in Fw, $10 \mathrm{Px}$ in $\mathrm{Hw}$.

Abdomen (Figure 5a). S1-8 dark brown dorsally, pale laterally, with pale apical rings, black color broadly extending on dorsal and lateral S8; S9 black/dark brown with a pale spot on its posterior dorsal $1 / 2$; S10 pale dorsally, black laterally; cerci black; ovipositor valves pale, surpassing posterior margin of $\mathrm{S} 10$.

Measurements. TL: 32.3; AB: 27.3; head width: 3; FW: 19.2; HW: 17.9; Pt: 0.6; metathoracic femur 2.3; metathoracic tibia 1.9.

Diagnosis. Similarly to the male, the female of F. machadorum can be easily separated from the other female congeners by the morphology of the prothoracic hind lobe. It presents a roughly squared hind prothoracic lobe (Figure 5d), whereas in F. sancta (Hagen in Selys, 1860), its closely related species, the hind lobe is rounded. Among
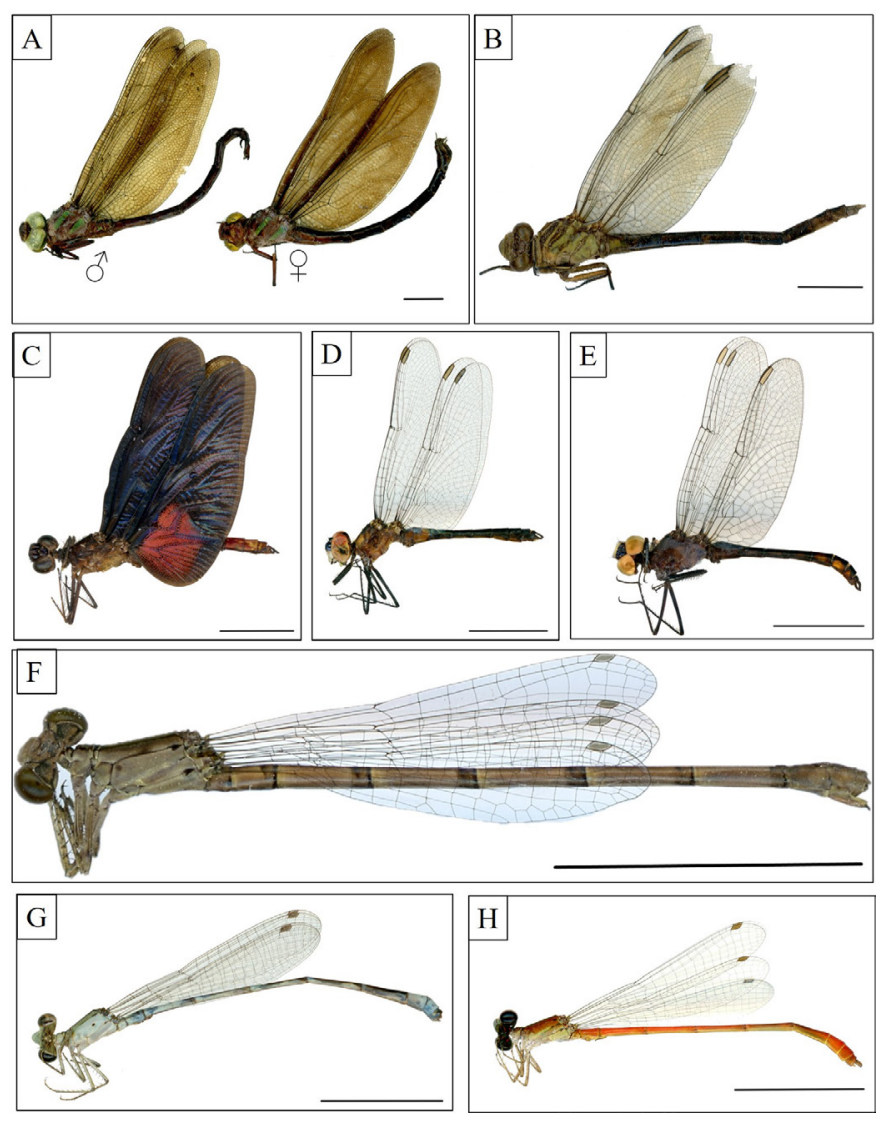

Figure 4. New species records for the state of Minas Gerais found in Uberlândia: a) male and female of Neuraeschna claviforcipata (Aeshnidae); b) female of Phyllocycla cf. medusa (Gomphidae); c) male of Diastatops intensa (Libellulidae); d) juvenile male of Oligoclada pachystigma (Libellulidae); e) male of Oligoclada xanthopleura (Libellulidae); f) female of Angelagrion nathaliae (Coenagrionidae); g) male of Telebasis simulacrum (Coenagrionidae); h) male of Telebasis sanguinalis (Coenagrionidae). Specimens were scanned at 1.200 dpi with the scanner Epson ${ }^{\circledR}$ V600 Perfection. Scale $=1 \mathrm{~cm}$.

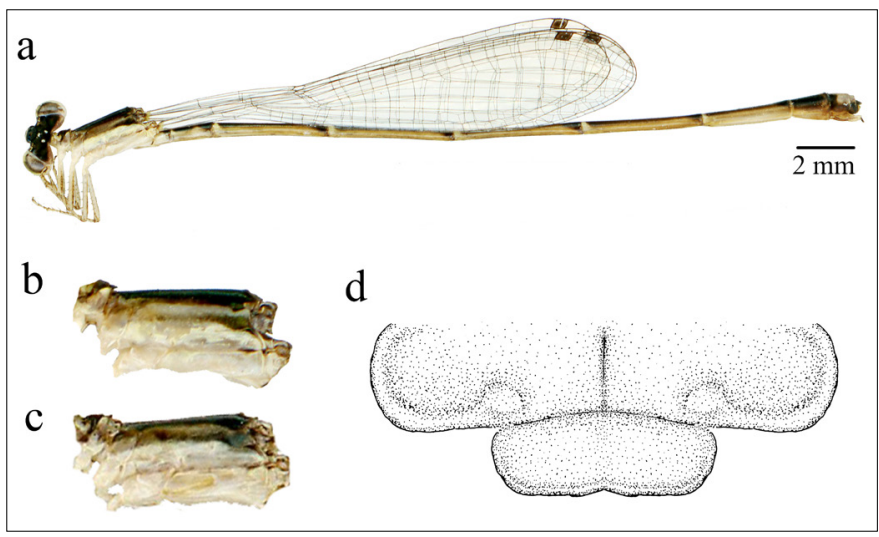

Figure 5. The female of Forcepsioneura machadorum Vilela, Venâncio \& Santos, 2020: (a) lateral view of habitus; (b-c) lateral close-up of thorax; (d) dorsal view of prothoracic hind lobe.

the examined females, the morphology is identical, although some coloration variation could be observed, as seen in Figure 4.

\section{Discussion}

Considering the knowledge gaps of Odonata in the Cerrado, we conducted an inventory in palm swamps and riparian forests of 
Uberlândia, Minas Gerais, in addition to the taxonomic description of the female of Forcepsioneura machadorum, and the description of the larvae of Acanthagrion truncatum (Vilela et al. 2020c). Altogether, we found 101 odonate species in the municipality, representing approximately $32 \%$ and $11 \%$ of the total richness in Minas Gerais and Brazil, respectively. Our study also added eight new records for the state, which now has 312 recorded species (Vilela 2020). This number represents the second highest Odonata richness reported to a Brazilian state, being only surpassed by Amazonas, with 335 species (Koroiva et al. 2020).

The number of species in palm swamp, riparian forest and both habitats, are greater in relation to standard diversity found in others inventories in the Cerrado, which, on average, are close to 50 species (Vilela et al. 2016, Barbosa et al. 2019, Borges et al. 2019). Possibly the heterogeneity of sampled sites can justify this difference. Other inventories with this pattern presented similar diversity to ours, even with fewer sampling effort. For example, in Minas Gerais, Bedê et al. (2015), Vilela et al. (2020a) and Amorim et al. (2018) sampled 128, 90 and 71 species, respectively, in more than 13 lotic and lentic sites. In other states, with less than 12 water bodies, Ferreira-Peruquetti \& Fonseca-Gessner (2003) registered 85 species in lotic and lentic sites of riparian forests of São Paulo, and Juen et al. (2014) found more than 2,000 specimens and 79 species in streams of three Cerrado phytophysiognomies in Mato Grosso. In addition, some extensive inventories of heterogeneous habitats in Cerrado and Atlantic Forest presented great numbers of new records for Minas Gerais (Bedê et al. 2015, Souza et al. 2017), which may also explain the novelties found in this study. Regardless of the comparisons, all surveys of dragonflies in the country are of great importance, since it contributes to Odonata database, ecology and taxonomy, even with small sampling effort.

The abundance and number of new records in our study were concentrated mainly on Libellulidae and Coenagrionidae. This result was expected, since these groups are represented by a great diversity of species present in numerous aquatic habitats in worldwide (Garrison et al. 2006, Garrison et al. 2010), and are commonly representative in Brazilian inventories (Souza et al. 2013, Calvão et al. 2014, Pires et al. 2019, Garcia Junior et al. 2021). Nevertheless, Aeshnidae and Gomphidae were slightly diverse in our study compared to other Cerrado inventories. This representativity may be justified due to larvae rearing and use of light traps methods, which, only in two sites, sampled almost $30 \%$ of the group diversity. Our results reinforce the adoption of alternative methods to sample these families, since adults of aeshnids and gomphids are strongfliers, cryptic and some species are crepuscular (Ferreira-Peruquetti \& Fonseca-Gessner 2003, Garrison et al. 2006, Almeida et al. 2013). Other families, such as Cordullidae and Megapodagrionidae were not recorded in our study. Despite nearly 50 or more species of these groups are known to occur in the neotropics (von Ellenrieder 2009), there are few inventoried species in Minas Gerais, with approximately 10 records in the Cerrado or ecotone zones of this biome and Atlantic Forest (Vilela 2020).

The riparian vegetation of many sampled sites were open with native grasses in palm swamp or, almost always, exotic grasses in disturbed forests. This may explain the great diversity of some libellulids, such as Erythrodiplax and Micrathyria. These groups are predominantly habitat generalists, abundant in open areas and some species are indicators of degraded sites, such as Erythrodiplax fusca Rambur, 1842 (Dutra \& De
Marco 2015, Dalzochio et al. 2018b), the most abundant libellulid in our study. However, even if anthropized areas are diverse in generalist dragonflies, they may contain not reported species with restricted distribution (Barbosa et al. 2019, Santos et al. 2020). In this context, we found Oligoclada xanthopleura and Angelagrion nathaliae in degraded ponds. The first species was not yet reported for the southeastern region, and the second occurred only in São Paulo state.

Despite the prevalence of generalist groups, few inventoried species and often restricted to integer habitats, such as Neuraeschna claviforcipata, Mnesarete guttifera Selys, 1873, Perithemis icteroptera Selys in Sagra, 1857 and Telebasis willinki Fraser, 1948 were found in the municipality. These records occurred mainly in urban green areas and conservation units, that even representing a small portion of the sampled locations, also held for approximately $50 \%$ of the novelties of this study. These results show that vegetation remnants hold a great richness of Odonata species, even being near or at urban areas. New species for science and states are often reported in protected urban or rural areas (e.g., Bedê et al. 2015, Pinto 2019), even if adjacent matrixes are unfavorable for odonates. For example, Erythrodiplax ana and $F$. machadorum were first discovered, respectively, at a preserved palm swamp and forest fragment adjacent to urban and cattle areas (Guillermo-Ferreira et al. 2016, Vilela et al. 2020b). Moreover, Acanthagrion marinae Lozano \& Rodrigues, 2018, a recent discovered coenagrionid, was also recorded in preserved urban palm swamps in Brazil (Lozano \& Rodrigues 2018, Rodrigues et al. 2019). In addition to provide suitable habitats to sensitive and low dispersion populations, most part of regional dragonfly community can also be benefited in conserved fragments, since these areas hold resources and microhabitats absent in degraded matrices (Ferreira-Peruquetti \& Fonseca-Gessner 2003, Paulson et al. 2006, Araújo et al. 2020). These benefits can extend to surrounding zones of low degradation level that contain some environmental characteristics of preserved fragments, being also favorable to sensitive species and different communities (MonteiroJúnior et al. 2016, Rodrigues et al. 2019). This highlights the importance of preserving and increase the number of such preserved areas and surrounding low disturbed zones to maintain riverine species (Oliveira et al. 2017, Azevedo-Santos et al. 2019). Despite this importance, the number of protected areas with incorporated aquatic environments is scarce in the Brazil, not ideally protecting most of aquatic taxa, including Odonata (Nóbrega \& De Marco 2011, Azevedo-Santos et al. 2019).

The IUCN red list is an important indicator of the conservation of global species for communities within and outside the scientific and conservation ambits (IUCN 2020). Considering some population characteristics, such as size, dynamics and geographic distribution (Rodrigues et al. 2006), several species are categorized under some conservation status (IUCN 2020). Nonetheless, data of some threatened groups, such as dragonflies, are still incomplete (Clausnitzer et al. 2009, Koroiva et al. 2017, Ávila Júnior et al. 2021). Our results showed that more than half of the species sampled in this study (including not evaluated species) do not present enough data to be listed in a threaten category, confirming the knowledge gap of Odonata for Brazil (Vilela et al. 2020b). With the increment of inventories and updates of vulnerability statuses in the future, many species restricted to a single habitat or that have been recently discovered will be included in some risk of extinction. For example, E. ana, a species restricted to preserved palm swamps (Guillermo-Ferreira et al. 2016, Vilela et al. 2020b), is 
listed at risk of extinction due to the low number of individuals, low geographic coverage and to the vulnerability of this phytophysiognomy. Thus, we believe that our present data will contribute to fill the gaps about the vulnerability of odonates. However, we emphasize again the need for more surveys of the group in palms swamps, riparian forests and other phytophysiognomies of Brazil.

All phytophysiognomies present unique species occurrence. Forest habitats had a higher diversity of exclusive damselflies, mainly composed by shade demand species (ex., Oxyagrion basale Selys, 1876 and Protoneura tenuis Selys, 1860), while palm swamps were diverse in open habitat dragonflies (ex., Micrathyria spp. and Erythemis spp.). This result indicates bank vegetation as an important regulator of local species for each habitat due to thermoregulatory demands of many Zygoptera and Anisoptera, as presented by many studies (De Marco et al. 2015, Carvalho et al. 2013, Oliveira-Júnior \& Juen 2019). Moreover, aquatic vegetation and abiotic factors are also related to Odonata structure of these habitats (Juen et al. 2007, Borges et al. 2021). For example, palm swamp and open habitats, such as degraded areas (Fares et al. 2020), are diverse in macrophytes, which are used as oviposition site or substrate during larvae development of some dependent libellulids and coenagrionids species (Vilela et al. 2016, Brito et al. 2020). Notably, degradation level, biased sample methods and time effort between phytophysiognomies must also be considered in our results, since some species found in a specific habitat in our study were present or absent in other palm swamp and forest studies (Rodrigues et al. 2018, Barbosa et al. 2019, Vilela et al. 2020a).

Here we demonstrated that inventories and taxonomic studies in poorly explored phytophysiognomies are important to overcome knowledge gaps of Brazilian odonatofauna. In addition to contributing to the knowledge of the diversity of dragonflies, new species reports, with other groups that inhabit palm swamps and forests, reinforce the importance of conserving these habitats, since many endemic species are at risk of extinction with land use expansion in these habitats. Therefore, we recommend more inventories of such phytophysiognomies in other regions.

\section{Acknowledgments}

We thank Afonso Galhardo, Guilherme Viana and Rodrigo Reis for support during fieldwork and Frederico A. A. Lencioni for the identification of $A$. nathaliae. We are also grateful to secretariat of the environment and urban services of Uberlândia, FUTEL, to Instituto Estadual de Florestas (IEF), Universidade Federal de Uberlândia (UFU) and to farms owners for access to urban parks, conservation units and rural properties. HV thanks Conselho Nacional de Desenvolvimento Científico e Tecnológico (CNPq) for a doctoral scholarship grant (\#140158/2018-9) and research productivity grant \#312752/2018-0 to JCS.

\section{Author Contributions}

Henrique Venâncio: Substantial contribution in the concept and design of the study. Contribution to data collection. Contribution to data analysis and interpretation. Contribution to manuscript preparation. Contribution to critical revision, adding intellectual content.

Diogo Silva Vilela: Substantial contribution in the concept and design of the study. Contribution to data collection. Contribution to data analysis and interpretation. Contribution to manuscript preparation. Contribution to critical revision, adding intellectual content.

Marcela Silva Barbosa: Contribution to data collection. Contribution to critical revision, adding intellectual content.
Jean Carlos Santos: Substantial contribution in the concept and design of the study. Contribution to data analysis and interpretation. Contribution to manuscript preparation. Contribution to critical revision, adding intellectual content.

\section{Conflicts of interest}

The authors declare that they have no conflict of interest related to the publication of this manuscript.

\section{References}

ALBERT, J.S., DESTOUNI, G., DUKE-SYLVESTER, S.M., MAGURRAN, A.E., OBERDORFF, T., REIS, R.E., WINEMILLER, K.O. \& RIPPLE, W.J. 2020. Scientists' warning to humanity on the freshwater biodiversity crisis. Ambio. 50:85-94.

ALMEIDA, M.V.O.D., PINTO, Â.P., CARVALHO, A.D.L. \& TAKIYA, D.M. 2013. When rare is just a matter of sampling: unexpected dominance of clubtail dragonflies (Odonata, Gomphidae) through different collecting methods at Parque Nacional da Serra do Cipó, Minas Gerais State, Brazil. Rev. Bras. Entomol. 57(4):417-423.

ALVARES, C.A., STAPE, J.L., SENTELHAS, P.C., MORAES GONÇALVES, J.L. \& SPAROVEK, G. 2013. Köppen's climate classification map for Brazil. Meteorol. Z. 22(6):711-728.

AMORIM, M.S., SOUZA, M.M. \& ANJOS, C.S. 2018. Riqueza de libélulas (Insecta: Odonata) no município de Bueno Brandão, sul de Minas Gerais. MG. Biota. 11(1):16-32.

ARAÚJO, M.F.A., DE MARCO, P., JUEN, L. \& TÔRRES, N.M. 2020. Vulnerability of Phyllocycla Species (Odonata: Gomphidae) to current and planned anthropic activities by the Brazilian Government. Neotrop. Entomol. 49(1):24-32.

ARAÚJO, G.M., BARBOSA, A.A., ARANTES, A.A. \& AMARAL, A.F. 2002. Composição florística de veredas no Município de Uberlândia, MG. Braz. J. Bot. 25(4):475-493.

ÁVILA JÚNIOR, W.F., MACHADO, G.L.V., LENCIONI, F.A.A. \& CARNEIRO, M.A.A. 2020. Distribution and composition of Dragonfly and Damselfly species (Odonata) of the upper Rio das Velhas, Ouro Preto, Minas Gerais State, Brazil. Pap. Avulsos Zool. 60:e20206065

AZEVEDO-SANTOS, V.M., FREDERICO, R.G., FAGUNDES, C.K., POMPEU, P.S., PELICICE, F.M., PADIAL, A.A., NOGUEIRA, M.G., FEARNSIDE, P.M., LIMA, L.B., DAGA, V.S., OLIVEIRA, F.J.M., VITULE, J.R.S., CALLISTO, M., AGOSTINHO, A.A., ESTEVES, F.A., LIMA-JUNIOR, D.P., MAGALHÃES, A.L.B., SABINO, J., MORMUL, R.P., GRASEL, D., ZUANON, J., VILELLA, F.S. \& HENRY, R. 2019. Protected areas: A focus on Brazilian freshwater biodiversity. Divers. Distrib. 25(3):442-448.

BARBOSA, M.S., BORGES, L.R., VILELA, D.S., VENÂNCIO, H. \& SANTOS, J.C. 2019. Odonate Communities of the Sucupira Reservoir, Rio Uberabinha, Minas Gerais, Brazil. Pap. Avulsos Zool. 59:e20195922.

BASTOS, R.C., BRASIL, L.S., CARVALHO, F.G., CALVÃO, L.B., SILVA, J.O.D.A. \& JUEN, L. 2019. Odonata of the state of Maranhão, Brazil: Wallacean shortfall and priority areas for faunistic inventories. Biota Neotrop. 19(4) e20190734. https://doi.org/10.1590/1676-0611-bn-2019-0734 (last access on 15/11/2020).

BEDÊ, L.C., MACHADO, A.B.M., PIPER, W. \& SOUZA, M.M. 2015. Odonata of the Serra de São José-Brazil's first Wildlife Reserve aimed at the conservation of dragonflies. Not. Odonatol. 8(5):117-155.

BELLE, J. 1988. A synopsis of the species of Phyllocycla Calvert, with descriptions of four new taxa and a key to the genera of Neotropical Gomphidae (Odonata, Gomphidae). Tijdschr. Entomol. 131:73-102.

BOLPAGNI, R., LAINI, A., STANZANI, C. \& CHIARUCCI, A. 2018. Aquatic plant diversity in Italy: Distribution, drivers and strategic conservation actions. Front. Plant. Sci. 9:e00116. 
BORGES, L.R., BARBOSA, M.S., CARNEIRO, M.A.A., VILELA, D.S. \& SANTOS, J.C. 2019. Dragonflies and damselflies (Insecta: Odonata) from a Cerrado area at Triângulo Mineiro, Minas Gerais, Brazil. Biota Neotrop. 19(1):e20180609. http://dx.doi.org/10.1590/1676-0611-bn-2018-0609 (last access on $22 / 10 / 2020$ ).

BORGES, L.R., BARBOSA, M.S., CARNEIRO, M.A.A. \& SANTOS, J.C. 2021. Habitat integrity drives Odonata diversity in Eucalyptus-dominated landscape. Environ. Monit. Assess. 193(1):1-14.

BRASIL, L.S., FERREIRA, V.R.S., RESENDE, B.O, JUEN, L., BATISTA, J.D., CASTRO, L.A. \& GIEHL, N.F.S. 2021. Dams Change Beta Diversity of Aquatic Communities in the Veredas of the Brazilian Cerrado. Front. Ecol. Evol. 9:40.

BRITO, J.S., MICHELAN, T.S. \& JUEN, L. 2020. Aquatic macrophytes are important substrates for Libellulidae (Odonata) larvae and adults. Limnology. 1-11.

CALVÃO, L.B., JUEN, L., OLIVEIRA-JÚNIOR, J.M.B., BATISTA, J.D. \& DE MARCO, P. 2018. Land use modifies Odonata diversity in streams of the Brazilian Cerrado. J. Insect Conserv. 22: 675-685.

CALVÃO, L.B., DE MARCO, P. \& BATISTA, J.D. 2014. List Odonata (Insecta) from Nova Xavantina, Mato Grosso, Central Brazil: Information on species distribution and new records. Check List. 10(2):299-307.

CALVÃO, L.B., NOGUEIRA, D.S., MONTAG, L.F.A., LOPES, M.A. \& JUEN, L. 2016. Are Odonata communities impacted by conventional or reduced impact logging? For. Ecol. Manag. 382:143-150.

CALVERT, P.P. 1909. Contributions to a knowledge of the Odonata of the Neotropical region, exclusive of Mexico and Central America. Ann. Carnegie Mus. 6(3):73-280

CLAUSNITZER, V., KALKMAN, V.J., RAM, M., COLLEN, B., BAILLIE, J.E.M., BEDJANIČ, M., DARWALL, W.R.T., DIJKSTRA, K.B., DOW, R., HAWKING, J., KARUBE, H., MALIKOVA, E., PAULSON, D., SCHÜTTE, K., SUHLING, F., VILLANUEVA, R.J., von ELLENRIEDER, N. \& WILSON, K., 2009.Odonata enter the biodiversity crisis debate: the first global assessment of an insect group. Biol. Conserv. 142:1864-1869.

CARDOSO, E. \& SCHIAVINI, I. 2002. Relação entre distribuição de espécies arbóreas e topografia em um gradiente florestal na Estação Ecológica do Panga (Uberlândia, MG). Rev. Bras. Bot. 25(3):277-289.

CARVALHO, F.G., PINTO, N.S., OLIVEIRA-JÚNIOR, J.M.B. \& JUEN, L. 2013. Effects of marginal vegetation removal on Odonata communities. Acta Limnol. Bras. 25:10-18.

CEZÁRIO, R.R., FIRME, P.P., PESTANA, G.C., VILELA, D.S., JUEN, L., CORDERO-RIVERA, A. \& GUILLERMO, R. 2020. Sampling Methods for Dragonflies and Damselflies. In Measuring Arthropod Biodiversity (J.C. Santos \& G.W. Fernandes, eds). Springer, Cham, p. 223-240.

CHAO, A., GOTELLI, N.J., HSIEH, T.C., SANDE, E.L., MA, K.H., COLWELL, R.K. \& Ellison, A.M. 2014. Rarefaction and extrapolation with Hill numbers: a framework for sampling and estimation in species diversity studies. Ecol. Monogr. 84:45-67.

COLLI, G.R., VIEIRA, C.R. \& DIANESE, J.C. 2020. Biodiversity and conservation of the Cerrado: recent advances and old challenges. Biodivers. Conserv. 29:1465-1475

CORBET, P.S. 1980. Biology of Odonata. Ann. Rev. Entomol. 25(1):189-217.

CORRÊA, C.C. 2014. Diversidade de libélulas em lagoas antrópicas: efeitos de área, isolamento e qualidade de hábitat. Dissertação de mestrado, Universidade Federal de Goiás, Goiânia.

COSTA, J.M., MACHADO, A.B.M., LENCIONI, F.A.A. \& SANTOS, T.C. 2000 Diversidade e distribuição dos Odonata (Insecta) no estado de São Paulo, Brasil: Parte I - Lista das espécies e registros bibliográficos. Publicações Avulsas do Mus. Nac. 80:1-27.

COSTA, J.M., PUJOL-LUZ, J. \& REGIS, L.L. 2004. Description of the larva of Zenithoptera anceps (Odonata, Libellulidae). Iheringia, Sér. Zool. 94(4):421-424.

DALZOCHIO, M.S., RENNER, S., SGANZERLA, C., PRASS, G., ELY, G.J., SALVI, L.C., DAMETTO, N. \& PÉRICO, E. 2018a. Checklist of Odonata (Insecta) in the state of Rio Grande do Sul, Brazil with seven new records. Biota Neotrop. 18(4):e20180551. http://dx.doi.org/10.1590/1676-0611- bn2018-0551 (last access on 03/10/2020)
DALZOCHIO, M.S. PÉRICO, E., RENNER, S. \& SAHLÉN, G. 2018b. Effect of tree plantations on the functional composition of Odonata species in the highlands of southern Brazil. Hydrobiologia. 808(1):283-300.

DE MARCO, P. \& VIANNA, D.M. 2005. Distribuição do esforço de coleta de Odonata no Brasil - subsídios para escolha de áreas prioritárias para levantamentos faunísticos. Lundiana 6:13-26.

DE MARCO, P., BATISTA, J.D. \& CABETTE, H.S.R. 2015. Community assembly of adult odonates in tropical streams: an ecophysiological hypothesis. PLoS One. 10(4):e0123023.

DUTRA, S. \& DE MARCO, P. 2015. Bionomic differences in odonates and their influence on the efficiency of indicator species of environmental quality. Ecol. Indic. 49:132-142.

FARES, A.L.B., CALVÃO, L.B., TORRES, N. R., GURGEL, E.S.C. \& MICHELAN, T.S. 2020. Environmental factors affect macrophyte diversity on Amazonian aquatic ecosystems inserted in an anthropogenic landscape. Ecol. Indic. 113:e106231.

FERREIRA-PERUQUETTI, P.S. \& FONSECA-GESSNER, A.A. 2003. Odonata community on natural areas of Cerrado and monoculture of northeastern São Paulo State, Brazil: relationship between land use and richness. Rev. Bras. Zool. 20(2):219-224.

GARCIA JUNIOR, M.D.N., DAMASCENO, M.T.S., MARTINS, M.J.L., COSTA, T.S., FERREIRA, R.M.A. \& SOUTO, R.N.P. 2021. New records of dragonflies and damselflies (Insecta: Odonata) from Amapá state, Brazil. Biota Neotrop. 21(1):e20201074. https://dx.doi.org/10.1590/1676-0611bn-2020-1074 (last access on 13/12/2020).

GARRISON, R.W. 2009. A synopsis of the genus Telebasis (Odonata: Coenagrionidae). Int. J. Odonatol. 12(1):1-121.

GARRISON, R.W., VON ELLENRIEDER, N. \& LOUTON, J.A. 2006. Dragonfly genera of the new world: an illustrated and annotated key to the Anisoptera. The John Hopkins University Press, Baltimore.

GARRISON, R.W., VON ELLENRIEDER, N. \& LOUTON, J.A. 2010. Damselfly genera of the new world: an illustrated and annotated key to the Zygoptera. The John Hopkins University Press Baltimore.

GUERRA, V., JARDIM, L., LLUSIA, D., MÁRQUEZ, R. \& BASTOS, R.P. 2020. Knowledge status and trends in description of amphibian species in Brazil. Ecol. Indic. 118:106754.

GUILLERMO-FERREIRA, R., VILELA, D.S., DEL-CLARO, K. \& BISPO, P.C. 2016. Erythrodiplax ana sp. nov. (Odonata: Libellulidae) from Brazilian palm swamps. Zootaxa, 4158(2):292-300

HECKMAN, C.W. 2006. Encyclopedia of South American aquatic insects: Odonata-Anisoptera: illustrated keys to known families, genera, and species in South America. Springer Science \& Business Media.

HORTAL, J., DE BELLO, F. DINIZ-FILHO, J.A.F., LEWINSOHN, T.M., LOBO, J.M. \& LADLE, R.J. 2015. Seven shortfalls that beset large-scale knowledge of biodiversity. Ann. Rev. Ecol. Evol. Syst. 46:523-549.

HSIEH, T.C., MA, K.H. \& CHAO, A. 2020. iNEXT: Interpolation and Extrapolation for Species Diversity. R package version 2.0.20. http://chao. stat.nthu.edu.tw/wordpress/software download/. (last access in 21/11/2020).

IBGE. 2020. https://cidades.ibge.gov.br/brasil/mg/uberlandia/panorama. (last access in 18/10/2020)

ICMBIO. 2018. Livro vermelho da fauna brasileira ameaçada de extinção. https://www.icmbio.gov.br/portal/images/stories/comunicacao/publicacoes/ publicacoes-diversas/livro_vermelho_2018_vol1.pdf(last access in 9/12/2020)

IUCN. 2020. IUCN Red List of Threatened species. https://www.iucnredlist. org/ (last access in 03/03/2021).

JUEN, L., CABETTE, H.S.R. \& DE MARCO, P. 2007. Odonate assemblage structure in relation to basin and aquatic habitat structure in Pantanal wetlands. Hydrobiologia. 579:125-134.

JUEN, L., OLIVEIRA-JÚNIOR, J.M.B., SHIMANO, Y., MENDES, T.P. \& CABETTE, H.S.R. 2014. Composição e riqueza de Odonata (Insecta) em riachos com diferentes níveis de conservação em um ecótone CerradoFloresta Amazônica. Acta Amaz. 44(2):223-233.

JUEN, L. \& De MARCO, P. 2012. Dragonfly endemism in the Brazilian Amazon: competing hypotheses for biogeographical patterns. Biodivers. Conserv. 21(13):3507-3521. 
KOROIVA, R., NEISS, U.G., FLECK, G. \& HAMADA, N. 2020. Checklist of dragonflies and damselflies (Insecta: Odonata) of the Amazonas state, Brazil. Biota Neotrop. 20(1):e20190877. http://doi.org/10.1590/1676-0611bn-2019-0877 (last access on 15/10/2020).

KOROIVA, R., RODRIGUES, M.E., VALENTE-NETO, F. \& ROQUE, F.O. 2017. Odonates from Bodoquena Plateau: checklist and information about endangered species. Biota Neotrop. 16(3):e20160310. http://dx.doi. org/10.1590/1676-0611-bn-2016-0310 (last access on 15/10/2020).

LATRUBESSE, E.M., ARIMA, E., FERREIRA, M.E., NOGUEIRA, S.H., WITTMANN, F., DIAS, M.S., DAGOST, F.C.P. \& BAYER, M. 2019. Fostering water resource governance and conservation in the Brazilian Cerrado biome. Conservation Science and Practice, 1(9):e77.

LENCIONI, F.A. 2008. Angelagrion gen. nov. with description of A. nathaliae sp. nov. and A. fredericoi sp. nov. from Brazil (Odonata: Coenagrionidae). Zootaxa. 1968(1):23-32.

LENCIONI, F.A.A. 1999. The genus Phasmoneura, with description of Forcepsioneura. gen. nov. and two new species (Zygoptera: Protoneuridae). Odonatologica. 28(2):127-137.

LENCIONI, F.A.A. 2017. Damselflies of Brazil - an illustrated identification guide - Southeast region. E-book.

LETSCH, H., GOTTSBERGER, B. \& WARE, J.L. 2016. Not going with the flow: a comprehensive time-calibrated phylogeny of dragonflies (Anisoptera: Odonata: Insecta) provides evidence for the role of lentic habitats on diversification. Mol. Ecol., 25(6):1340-1353.

LOZANO, F. \& RODRIGUES, M.E. 2018. Acanthagrion marinae sp. nov. (Zygoptera: Coenagrionidae): a new species of the apicale group. An. Acad. Bras. Ciênc. 90(3):2865-2872.

MAYWALD, P.G. \& MARÇAL-JÚNIOR, O. 2013. Estrutura de Áreas protegidas dos assentamentos de reforma agrária no município de Uberlândia-MG, Brasil: um estudo de ecologia de paisagem. Soc. Nat. 25(1):75-90.

MCKINNEY, M.L. 2002. Urbanization, Biodiversity, and Conservation: The impacts of urbanization on native species are poorly studied, but educating a highly urbanized human population about these impacts can greatly improve species conservation in all ecosystems. Bioscience. 52(10):883-890.

Miguel, T.B., CALVÃO, L.B., VITAL, M.V.C. \& JUEN, L. 2017. A scientometric study of the order Odonata with special attention to Brazil. Int. J. Odonatol. 20: 27-42.

MONTEIRO-JÚNIOR, C., ESPOSITO, M.C. \& JUEN, L. 2016. Are the adult odonate species found in a protected area different from those present in the surrounding zone? A case study from eastern Amazonia. J. Insect Conserv. 20(4):643-652.

MYERS, N., MITTERMEIER, R.A., MITTERMEIER, C.G., FONSECA, G.A.B. \& KENT, J. 2000. Biodiversity hotspots for conservation priorities. Nature (403):853-858.

NEEDHAM, J.G., WESTFALL JR, M.J. \& MAY, M.L. 2000. Dragonflies of North America (No. Rev. Ed). Scientific Publishers, Inc.

NETTO, F.M.L., DANELON, J.R.B. \& RODRIGUES, S.C. 2011. Evaluation of water quality and land uses of drainage basin of Terra Branca Stream Uberlandia - MG. Rev. Geogr. Acadêmica. 5(2):66-75.

NÓBREGA, C.C. \& DE MARCO, P. 2011. Unprotecting the rare species: a niche-based gap analysis for odonates in a core Cerrado area. Divers. Distrib. 17(3):491-505.

OLIVEIRA, U., SOARES-FILHO, B.S., PAGLIA, A.P., BRESCOVIT, A.D., CARVALHO, C.J.B., SILVA, D.P., REZENDE, D.T., FORTES-LEITE, F.S., BATISTA, J.A.N., BARBOSA, J.P.P.P., STEMANN, J.R., ASCHER, J.S., DE MARCO, P., LÖWENBERG-NETO, P., GIANLUPPI FERRO, V. \& SANTOS, A.J. 2017. Biodiversity conservation gaps in the Brazilian protected areas. Sci. Rep. 7(1):9141.

OLIVEIRA-FILHO, A.T. \& RATTER, J.A. 2002. Vegetation physiognomies and woody flora of the cerrado biome. In The cerrados of Brazil: ecology and natural history of a neotropical savanna (P.S. Oliveira \& R.J. Marquis, eds.). Columbia University Press, New York, p.91-120.

OLIVEIRA-JÚNIOR, J.M.B. \& JUEN, L. 2019. The Zygoptera/Anisoptera ratio (Insecta: Odonata): a new tool for habitat alterations assessment in Amazonian streams. Neotrop. Entomol. 48(4):552-560.
PALACIO, A.D., MUZON, J., JUEN, L., FERREIRA, V.R.S. \& BATISTA, J. D. 2020. Erythrodiplax nataliae sp. nov., a new species for the state of Mato Grosso, Brazil. An. Acad. Bras. Ciênc. 92(2):e20181149.

PAULSON, D. \& SCHORR, M. 2020. World Odonata List. Electronic Database. https://www.pugetsound.edu/academics/academic-resources/slater-museum/ biodiversity-resources/dragonflies/world-odonata-list2/ (last access in $7 / 10 / 2020)$

PAULSON, D. 2006. The importance of forests to neotropical dragonflies. Forests and dragonflies: Fourth WDA International Symposium of Odonatology (A. Rivera, ed.). Pontevedra, Spain, p.79-101.

PINTO, Â.P. \& LAMAS, C.J. 2011. Oligoclada mortis sp. nov. from Rondônia State, Brazil, and distributional records of other species of the genus (Odonata: Libellulidae). Int. J. Odonatol. 14(4):291-303.

PINTO, Â.P. \& CARVALHO, A.L. 2012. Taxonomic and distributional notes on Telebasis Selys, 1865, with a redescription of T. pallida Machado, 2010 , and an evaluation of the T. racenisi Bick \& Bick, 1995 "complex" of species (Odonata, Coenagrionidae). Dtsch. Ent. Z. 59(2):189-200.

PINTO, Â.P. 2019. First report on the dragonflies from Parque Estadual da Ilha do Cardoso, state of São Paulo, Brazil, with notes on the morphology and behavior of Lauromacromia picinguaba (Odonata: Corduliidae sl.). Stud. Neotrop. Fauna Environ. 54(1):48-60.

PINTO, Â.P. 2020. Odonata in Catálogo Taxonômico da Fauna do Brasil. PNUD. http://fauna.jbrj.gov.br/fauna/faunadobrasil/171 (last access on 9/10/2020)

PIRES, M.M., KOTZIAN, C.B., SGANZERLA, C., PRASS, G., DALZOCHIO, M.S. \& PÉRICO, E. 2019. Diversity of Odonata (Insecta) in Seasonal Deciduous Forest fragments in southern Brazil (state of Rio Grande do Sul), with a new record for the state and comments on the seasonal distribution of the species. Biota Neotrop. 19(4):e20190769. http://dx.doi. org/10.1590/1676-0611-bn-2019-0769. (last access on 23/11/2020)

R CORE TEAM. 2020. R: A language and environment for statistical computing. R Foundation for Statistical Computing, Vienna, Austria. URL https:// www.R-project.org/. (last access on 10/12/2020)

RATTER, J.A., RIBEIRO, J.F. \& BRIDGEWATER, S. 1997. The Brazilian cerrado vegetation and threats to its biodiversity. Ann. Bot. 80(3):223-230.

REID, A.J., CARLSON, A.K., CREED, I.F., ELIASON, E.J., GELL, P.A., JOHNSON, P.T., KIDD, K.A., MACCORMACK, T.J., OLDEN, J.D., ORMEROD, S.J., SMOL, J.P., TAYLOR, W.W., TOCKNER, K., VERMAIRE, J.C., DUDGEON, D. \& COOKE, S.J. 2019. Emerging threats and persistent conservation challenges for freshwater biodiversity. Biol. Rev. 94(3):849-873.

RENNER, S., PÉRICO, E., DALZOCHIO, M.S. \& SAHLÉN, G. 2018. Water body type and land cover shape the dragonfly communities (Odonata) in the Pampa biome, Rio Grande do Sul, Brazil. J. Insect Conserv. 22(1):113-125.

RIBEIRO, J.F. \& WALTER, B.M.T. 1998. As principais fitofisionomias do bioma Cerrado. In Cerrado ecologia e flora Vol 1. (S.M. Sano, S.P. Almeida \& J.F. Ribeiro, eds). Embrapa Cerrados, p.151-212.

RODRIGUES, A.S., PILGRIM, J.D., LAMOREUX, J.F., HOFFMANN, M. \& BROOKS, T. M. 2006. The value of the IUCN Red List for conservation. Trends Ecol. Evol. 21(2):71-76.

RODRIGUES, M.E. \& ROQUE, F.D.O. 2017. Checklist de Odonata do estado de Mato Grosso do Sul, Brasil. Iheringia, Sér. Zool. 107:107-111.

RODRIGUES, M.E., MOURA, E.B., KOROIVA, R., BORGES, A.C.P. \& OLIVEIRA-ROQUE, F. 2018. Survey of dragonflies (Odonata) in palm swamps of Cerrado hotspot. Entomol. News, 128(1):24-38.

RODRIGUES, M.E., MOURA, E.B. \& ROQUE, F. O. 2019. Dragonflies as indicators of the environmental conditions of veredas in a region of centralwestern Brazil. Oecologia Aust. 23(4):969-978.

ROSA, R.M., OLIVEIRA-FERREIRA, V. \& BRITO, J.L.S. 2019. Mapa de reconhecimento geológico da bacia do rio Uberabinha (MG): procedimento experimental utilizando coeficientes de concordância. Caminhos de Geografia. 20(70):507-518.

SÁNCHEZ-BAYO, F. \& WYCKHUYS, K.A. 2019. Worldwide decline of the entomofauna: A review of its drivers. Biol. Conserv. 232:8-27.

SANO, E.E., ROSA, R., BRITO, J.L. \& FERREIRA, L.G. 2010. Land cover mapping of the tropical savanna region in Brazil. Environ. Monit. Assess. 166(1):113-124. 
Venâncio, H. et al

SANTOS, J.C., VILELA, D.S., ALMEIDA, B.S., SANTOS, A.E., BEZERRA, L.M.M., SANTOS, L., NETO, A.M.S., VENÂNCIO, H. \& CARNEIRO, M.A.A. 2020. A rapid survey of dragonflies and damselflies (Insecta: Odonata) reveals 29 new records to Sergipe State, Brazil. Hetaerina. 2(2):29-34.

SGANZERLA, C., DALZOCHIO, M.S., PRASS, G.S. \& PÉRICO, E. 2021. Effects of urbanization on the fauna of Odonata on the coast of southern Brazil. Biota Neotrop. 21(1):e20201122. https://dx.doi.org/10.1590/16760611-bn-2020-1122. (last access on 02/03/2021).

SILVEIRA, O.T. 2003. Fauna de insetos das ressacas das bacias do Igarapé da Fortaleza e do Rio Curiaú. In Diagnóstico das ressacas do Estado do Amapá: bacias do Igarapé da Fortaleza e Rio Curiaú (L.R., Takiyama, A.Q., Silva, W.J.P., Costa, H.S, Nascimento, eds.). Macapá, AP, p.73-80.

SOUZA, M.M., SOUZA, B., AGUIAR PEREIRA, M.C.S. \& MACHADO, A.B.M. 2013. List of Odonates from Mata do Baú, Barroso, Minas Gerais, Brazil. Check List. 9(6):1367-1370.

SOUZA, M.M., PIRES, E.P., BRUNISMANN, Â.G., MILANI, L.R. \& PINTO, Â.P. 2017. Dragonflies and damselflies (Odonata) from the wetland of the Rio Pandeiros, northern region of Minas Gerais State, Brazil, with a description of the male of Archaeogomphus vanbrinki Machado (Anisoptera: Gomphidae). Int. J. Odonatol. 20(1):13-26.

THE GIMP DEVELOPMENT TEAM. 2019. GIMP. https://www.gimp.org (last access in 13/12/2020)

TROIA, M.J. \& MCMANAMAY, R.A. 2017. Completeness and coverage of open-access freshwater fish distribution data in the United States. Divers. Distrib. 23(12):1482-1498.

VELAZCO, S.J.E., VILLALOBOS, F., GALVÃO, F. \& DE MARCO, P. 2019. A dark scenario for Cerrado plant species: Effects of future climate, land use and protected areas ineffectiveness. Divers. Distrib. 25(4):660-673.

VERAS, D.S. 2017. Efeito da perda de integridade de habitat de riachos sobre as assembleias de Odonata (Insecta) na zona ecotonal do leste do Maranhão. Dissertação de mestrado. Universidade Estadual do Maranhão, Caxias.
VILELA, D.S., FERREIRA, R.G. \& DEL-CLARO, K. 2016. The odonatan community of a brazilian vereda: seasonal patterns, species diversity and rarity in a palm swamp environment. Biosci. J. 32(1):486-495.

VILELA, D.S., GUILLERMO-FERREIRA, R., DEL-CLARO, K. \& CORDERO-RIVERA, A. 2018. Argia angelae (Odonata: Zygoptera: Coenagrionidae) sp. nov. from Chapada dos Guimarães, Mato Grosso, Brazil. Zootaxa 4415(3):549-560.

VILELA, D.S. 2020. Libélulas de Minas Gerais. http://libelulasdemg.com.br/. (last access in 12/12/2020)

VILELA, D.S., KOROIVA, R., TOSTA, T.H.A., NOVAES, M.C. \& GUILLERMOFERREIRA, R. 2020a. Dragonflies and damselflies from the West of Minas Gerais, Brazil: checklist and new records. Biota Neotrop. 20(1): e20190851. http://dx.doi.org/10.1590/1676-0611-bn-2019-0851. (last access on 15/11/2020)

VILELA, D.S., VENÂNCIO, H. \& SANTOS, J.C. 2020b. Forcepsioneura machadorum (Coenagrionidae: Protoneurinae) sp. nov. from the Cerrado Biome of Minas Gerais, southeastern Brazil. Int. J. Odonatol. 23(4):397-404.

VILELA, D. S., VENÂNCIO, H. \& SANTOS, J. C. 2020c. Final instar larva of Acanthagrion truncatum Selys, 1876 (Zygoptera: Coenagrionidae). Zootaxa. 4881(2):393-400.

Von ELLENRIEDER, N. 2009. Databasing dragonflies: State of knowledge in the Neotropical region. Agrion. 13(2):58-72.

WEN, Y., SCHOUPS, G. \& van de GIESEN, N. 2017. Organic pollution of rivers: Combined threats of urbanization, livestock farming and global climate change. Sci. Rep. 7:e43289.

Received: 29/12/2020

Revised: 01/04/2021

Accepted: 06/04/2021

Published online: 12/05/2021 